\title{
DIFFERENTIAL RESOLVENTS OF MINIMAL ORDER AND WEIGHT
}

\author{
JOHN MICHAEL NAHAY
}

Received 10 February 2004

\begin{abstract}
We will determine the number of powers of $\alpha$ that appear with nonzero coefficient in an $\alpha$-power linear differential resolvent of smallest possible order of a univariate polynomial $P(t)$ whose coefficients lie in an ordinary differential field and whose distinct roots are differentially independent over constants. We will then give an upper bound on the weight of an $\alpha$-resolvent of smallest possible weight. We will then compute the indicial equation, apparent singularities, and Wronskian of the Cockle $\alpha$-resolvent of a trinomial and finish with a related determinantal formula.
\end{abstract}

2000 Mathematics Subject Classification: 12H05, 13 N15.

1. Introduction. It is the purpose of this paper to prove two theorems giving, respectively, a lower bound (Theorem 8.3) on the number of nonzero terms (more precisely called coefficient-functions in Definition 6.2) which must appear in a linear differential resolvent and an upper bound (Theorem 9.1) on the smallest possible weight of a resolvent for polynomials whose distinct roots are differentially independent over constants. Such polynomials are called differentially transcendental polynomials. They may be differentially specialized, meaning if $z \rightarrow \phi(z)$, then $D z \rightarrow \phi(D z)$, to any polynomial whose roots have the same multiplicities. Therefore, it is reasonable to begin the investigation of the bounds on the weight of and number of nonzero terms in resolvents for arbitrary polynomials with the investigation of these bounds on resolvents of differentially transcendental polynomials.

A differential $\alpha$-resolvent of a polynomial is itself a polynomial in $\alpha$. The author first recognized this fact by examining the partial differential equations of Mellin [16], who chose the letter $\alpha$. The author's powersum formula [21] for computing resolvents relies on the fact that an $\alpha$-resolvent of a polynomial is itself a polynomial in $\alpha$. The author asserts in [19, Theorem 4.1] that the powersum formula for $\alpha$-resolvents does not vanish identically for differentially transcendental polynomials.

In Theorem 8.3 we will determine the number of nonzero coefficients of $\alpha$ in an $n$ thorder $\alpha$-resolvent of a differentially transcendental polynomial with $n$ distinct roots. The author calls these coefficients of $\alpha$ the coefficient-functions of the resolvent to suggest that they are not necessarily constant. In Theorem 9.1 we will determine an upper bound on the weight, given by Definition 5.1, of these coefficient-functions for any integral $\alpha$-resolvent. It is important to determine the upper bound on the weight of a particular resolvent in [17] in order to assess how much factoring must be done.

2. Definitions and notation. Let $\mathbb{Z}$ denote the integers, let $\mathbb{N}$ denote the positive integers, let $\mathbb{N}_{0}$ denote the nonnegative integers, let $\mathbb{Q}$ denote the rational numbers. For any ring $\mathbb{R}$, let $\mathbb{R}^{\#}$ denote the nonzero elements of $\mathbb{R}$. 
Definition 2.1. For each $m \in \mathbb{N}$, define $[m] \equiv\{k \in \mathbb{N} \ni 1 \leq k \leq m\}$.

DEFINITION 2.2. For each $m \in \mathbb{N}_{0}$, define $[m]_{0} \equiv\left\{k \in \mathbb{N}_{0} \ni 0 \leq k \leq m\right\}$.

DEFINITION 2.3. For any variable or differential operator $v$ and any $m \in \mathbb{N}$ and $r \in \mathbb{Q}$, define

$$
(v)_{m, r} \equiv \prod_{k=1}^{m}(v-(k-1) \cdot r), \quad(v)_{0, r} \equiv 1,(v)_{m} \equiv(v)_{m, 1} .
$$

Definition 2.3 will be used extensively in the proof of Theorem 11.9.

Definition 2.4. Define the function $\delta_{i, j} \equiv 1$ if $i=j$ and $\delta_{i, j} \equiv 0$ if $i \neq j$. Denote a square matrix $\mathrm{M}$ whose entry in the $i$ th row and $j$ th column is some function $\mathrm{M}(i, j)$ of $i$ and $j$ by $[\mathrm{M}(i, j)]_{i \times j}$ and its determinant by $\operatorname{det}[\mathrm{M}(i, j)]_{i \times j}$.

We will follow the notation in [13] for the adjunction of differential elements to differential rings and fields. Let $\Theta$ denote an element or a set of elements in a differential ring extension of a differential ring or field $\mathbb{R}$. Brackets $\mathbb{R}[\Theta]$ will denote the polynomial ring generated by $\Theta$ over $\mathbb{R}$. Braces $\mathbb{R}\{\Theta\}$ will denote the differential ring generated by $\Theta$ over $\mathbb{R}$, which is just the polynomial ring generated by infinitely many derivatives of $\Theta$ over $\mathbb{R}$. Only when $\mathbb{R}$ is a field, parentheses $\mathbb{R}(\Theta)$ will denote the field generated by $\Theta$ over $\mathbb{R}$. Only when $\mathbb{R}$ is a differential field, arrows $\mathbb{R}\langle\Theta\rangle$ will denote the differential field generated by $\Theta$ over $\mathbb{R}$, which is just the field generated by infinitely many derivatives of $\Theta$ over $\mathbb{R}$. Finally, with a slight addition to Kolchin's notation, for each $m \in \mathbb{N}_{0}$, let $\mathbb{R}\{\Theta\}_{m}$ denote the ordinary (nondifferential) ring generated by $\mathbb{R}$ and the first $m$ derivatives of $\Theta$. Only when $\mathbb{R}$ is a field, let $\mathbb{R}\langle\Theta\rangle_{m}$ denote the ordinary (nondifferential) field generated by $\mathbb{R}$ and the first $m$ derivatives of $\Theta$. We remind the reader that differential rings and fields must contain infinitely many derivatives of all their elements.

We always have the following four set-theoretic inclusions and two equalities:

$$
\begin{array}{rlrl}
\mathbb{R}\{\Theta\}_{m} \subset \mathbb{R}\{\Theta\}, & \mathbb{R}\langle\Theta\rangle_{m} \subset \mathbb{R}\langle\Theta\rangle, & \mathbb{R}\{\Theta\}_{m} \subset \mathbb{R}\langle\Theta\rangle_{m}, & \mathbb{R}\{\Theta\} \subset \mathbb{R}\langle\Theta\rangle, \\
\mathbb{R}\{\Theta\}_{0}=\mathbb{R}[\Theta], & \mathbb{R}\langle\Theta\rangle_{0}=\mathbb{R}(\Theta) . &
\end{array}
$$

DEFINITION 2.5. Let $P(t) \equiv \sum_{i=0}^{N}(-1)^{N-i} e_{N-i} \cdot t^{i}=\prod_{i=1}^{n}\left(t-z_{i}\right)^{\pi_{i}} \in \mathbb{F}[t]$ be a monic univariate polynomial in $t$ of degree $N=\sum_{i=1}^{n} \pi_{i}$ with $n$ distinct roots $\left\{z_{i}\right\}_{i=1}^{n}$ whose coefficients $e \equiv\left\{e_{i}\right\}_{i=1}^{N}$ lie in an ordinary differential field $\mathbb{F}$ with derivation $D$.

Thus the $e$ are the first $N$ elementary symmetric functions of the roots of $P$ counting their multiplicities $\left\{\pi_{i}\right\}_{i=1}^{n}$. Henceforth, we will consider only the various differential subfields of $\mathbb{F}=\mathbb{Q}\left\langle z_{1}, \ldots, z_{n}\right\rangle(\alpha)$. Let $\left\{f_{i}\right\}_{i=1}^{r}$ be the irreducible factors of $P$ over the base field $\mathbb{Q}(e)$, also called the coefficient field of $P$. Then $P(t)=\prod_{i=1}^{r} f_{i}^{\tau_{i}}$, where $\left\{\boldsymbol{\tau}_{i}\right\}_{i=1}^{r} \subset\left\{\pi_{i}\right\}_{i=1}^{n}$. We call $\mathbb{Q}\langle e\rangle_{m}$ the $m$ th order coefficient field of $P, \mathbb{Q}\langle e\rangle$ the differential coefficient field of $P, \mathbb{Z}[e]$ the coefficient ring of $P, \mathbb{Z}\{e\}_{m}$ the $m$ th order coefficient ring of $P$, and $\mathbb{Z}\{e\}$ the differential coefficient ring of $P$.

DEFINITION 2.6. Define the monic univariate polynomial $\bar{P}(t) \equiv \prod_{i=1}^{n}\left(t-z_{i}\right)=$ $\sum_{i=0}^{n}(-1)^{n-i} \bar{e}_{n-i} \cdot t^{i}$ with the same roots as $P$ but all simple. 
Thus the coefficients $\bar{e} \equiv\left\{\bar{e}_{i}\right\}_{i=1}^{n}$ of $t^{i}$ in $\bar{P}$ in Definition 2.6 are the first $n$ elementary symmetric functions of the roots of $P$ not counting their multiplicities. Since $\operatorname{char}(\mathbb{F})=0$, $\mathbb{F}$ is a perfect field, so $P$ is a separable polynomial, so each of the irreducible factors of $P$ has simple roots, so $\bar{P}(t)=\prod_{i=1}^{r} f_{i}$. Since there exist only finitely many nonzero elementary symmetric functions of the roots of $P$, with or without multiplicities, it is understood that adjunction of $e$ and their derivatives means adjunction of the $N$ elements $e_{1}, \ldots, e_{N}$, and adjunction of $\bar{e}$ and their derivatives means adjunction of the $n$ elements $\bar{e}_{1}, \ldots, \bar{e}_{n}$. Thus, the definitions of $\mathbb{Q}(\bar{e}), \mathbb{Q}\langle\bar{e}\rangle_{m}, \mathbb{Q}\langle\bar{e}\rangle, \mathbb{Z}[\bar{e}], \mathbb{Z}\{\bar{e}\}_{m}$, and $\mathbb{Z}\{\bar{e}\}$ are clear. In contrast, there are infinitely many nonzero powersums (see formulae (4.1)(4.4)) and Schur functions (see Definition 4.2). So, when we wish to adjoin finitely many powersums to a field, we must specify which powersums we are adjoining.

DEFInITION 2.7. Define $\Delta \equiv \prod_{i<j}\left(z_{i}-z_{j}\right)^{2}$ to be the discriminant of $\bar{P}$. Note that $\Delta \neq 0$ and $\Delta$ lies in $\mathbb{Z}[\bar{e}]$.

LeMmA 2.8. Let $e$ and $\bar{e}$ be given by Definitions 2.5 and 2.6. Then the inclusion $\mathbb{Q}\langle\bar{e}\rangle_{m} \subset \mathbb{Q}\langle e\rangle_{m}$ holds.

Proof. If $P(t)=\prod_{i=1}^{r}\left(f_{i}(t)\right)^{T_{i}}$ is the irreducible factorization of $P(t)$ over $\mathbb{Q}(e)$, then each $f_{i}(t)$ lies in $\mathbb{Q}(e)[t]$. So $\bar{P}(t)=\prod_{i=1}^{r} f_{i}(t) \in \mathbb{Q}(e)[t]$. Since $\left\{\bar{e}_{i}\right\}_{i=1}^{n}$ are defined by $\bar{P}(t)=\sum_{i=0}^{n}(-1)^{n-i} \bar{e}_{n-i} \cdot t^{i}$, it follows that $\bar{e} \subset \mathbb{Q}(e)$. Hence $\mathbb{Q}\langle\bar{e}\rangle_{m} \subset \mathbb{Q}\langle e\rangle_{m}$.

No immediate inclusion like the inclusion in Lemma 2.8 is possible between the rings $\mathbb{Z}\{\bar{e}\}_{m}$ and $\mathbb{Z}\{e\}_{m}$. However, Lemma 4.3 gives us a partial inclusion.

DEFINITION 2.9. Let $\alpha$ be transcendental over $\mathbb{Q}\langle e\rangle$ with $D \alpha=0$. For each root $z$, let $y$ denote a nonzero solution of $z \cdot D y-\alpha \cdot y \cdot D z=0$. Call $y$ an $\alpha$-power of the root $z$ of $P$ and denote it by $y=z^{\alpha}$. Thus, $y$ is unique up to a constant multiple, and a unique $z$ corresponds to each $y$.

DEFINITION 2.10. Let a maximum of $o$ of the $\alpha$-powers of the roots be linearly independent over constants. Order the roots such that $\left\{y_{i}=z_{i}^{\alpha}\right\}_{i=1}^{o}$ are linearly independent over constants.

3. Partitions of integers. In this section we will use the definitions and some of the notation as in [14, page 1]. We must use the letters $i$ and $m$ elsewhere in this paper, so, in place of these letters in Macdonald's definitions, we will use the letters $k$ and $r$.

DEFINITION 3.1. For our purposes, a partition $\lambda$ is a finite decreasing sequence of nonnegative integers $\lambda_{1} \geq \lambda_{2} \geq \cdots \geq \lambda_{\ell}$, called the parts of $\lambda$.

Definition 3.2. The number of positive parts $\ell$ is the length of $\lambda$. To emphasize the particular partition, write $\ell(\lambda)$ for the length of $\lambda$.

DEFINITION 3.3. For each $k \in[n]$, define $r_{k} \in \mathbb{N}_{0}$ to be the number of parts of $\lambda$ equal to $k$. Call $r_{k}$ the multiplicity of $k$ in $\lambda$.

It is convenient not to deal directly with the individual parts $\lambda_{v}$ of a partition $\lambda$, but rather with these multiplicities and write $\lambda=\left(1^{r_{1}} 2^{r_{2}} \cdots n^{r_{n}}\right)$. Hence $\ell=\sum_{k=1}^{n} r_{k}$. 
DEFINITION 3.4. Define $|\lambda|$ to be the sum of the parts of $\lambda$ and call it the weight of $\lambda$. Hence, $|\lambda|=\sum_{k=1}^{n} k \cdot r_{k}$. Call $\lambda$ is a partition of the integer $|\lambda|$. Extend Macdonald's definitions slightly to allow zero parts and to define $\lambda=(0)$ to be the unique partition of zero.

We will use partitions of integers in two ways. In the first way in Section 4 and Lemma 4.3 in particular the $k$ th part $\lambda_{k}$ of the partition $\lambda$ is the power of $z_{k}$. Thus $r_{k}=0$ for all $k>n$ since all parts of $\lambda$ are less than or equal to $n$. So $\ell(\lambda) \leq n$. The weight $w\left(\bar{s}_{\lambda}\right)$ of the Schur polynomial $\bar{s}_{\lambda}$, which is the common notion of isobaric weight or total degree given by Definition 5.1, equals $|\lambda|$.

In the second way in Section 7 and formula (7.2) in particular the multiplicity $r_{k}$ is the power of the $k$ th derivative of $z_{l}$, so $|\lambda|=m$, the total differential order of the Bell polynomial $B_{m, k}\left\{z_{l}\right\}$, and the length $\ell(\lambda)$ of $\lambda$ equals the weight of $w\left(B_{m, k}\left\{z_{l}\right\}\right)$ given by Definition 5.1. Although we will need to consider only $m \leq n$ in Theorem 9.1 , one can differentiate infinitely many times, so in principle there is no upper bound on $m$, and therefore no upper bound exists on $|\lambda|$ and $\ell(\lambda)$ in this application of partitions of integers.

From Definitions 3.1, 3.2, 3.3, and 3.4, it follows that $1 \leq \ell(\lambda) \leq m$ for all partitions $\lambda$ of a positive integer $m$. Note the following two extreme cases on $\ell(\lambda)$.

ASSERTION 3.5. There exists exactly one partition $\lambda$ such that $\ell(\lambda)=1$, namely, $\lambda=\left(m^{1}\right)$. Thus $r_{m}=1$ and $r_{k}=0$ for all $k \neq m$.

ASSERTION 3.6. There exists exactly one partition $\lambda$ such that $\ell(\lambda)=m$, namely, $\lambda=\left(1^{m}\right)$. Thus $r_{1}=m$ and $r_{k}=0$ for all $k \neq 1$.

\section{Powersums}

DEFINITION 4.1. Define the $q$ th powersum of the roots of $P$ as $p_{q} \equiv \sum_{j=1}^{n} \pi_{j} \cdot z_{j}^{q}$, for all $q \in \mathbb{Z}$.

It is well-known that $p_{q} \in \mathbb{Z}\left[e_{1}, \ldots, e_{\min (q, N)}\right]$, for all $q \in \mathbb{N}_{0}$, and $e_{q} \in \mathbb{Q}\left[p_{1}, \ldots, p_{\min (q, N)}\right]$, for all $q \in \mathbb{N}_{0}$, with formulae given by [14, page 28]

$$
\begin{aligned}
& p_{q}=\operatorname{det}\left[\begin{array}{cccc}
e_{1} & 1 & 0 & 0 \\
2 e_{2} & e_{1} & \ddots & 0 \\
\vdots & \vdots & \ddots & 1 \\
q \cdot e_{q} & e_{q-1} & \cdots & e_{1}
\end{array}\right] \quad \forall q \in \mathbb{N} \text {, where } e_{q} \equiv 0, \forall q>N, \\
& e_{q}=\frac{1}{q !} \operatorname{det}\left[\begin{array}{cccc}
p_{1} & 1 & 0 & 0 \\
p_{2} & p_{1} & \ddots & 0 \\
\vdots & \vdots & \ddots & q-1 \\
p_{q} & p_{q-1} & \cdots & p_{1}
\end{array}\right] \quad \forall q \in \mathbb{N} .
\end{aligned}
$$


When $q>N$, formula (4.2) yields zero for $e_{q}$. For reference, we note that

$$
\begin{gathered}
\bar{p}_{q}=\operatorname{det}\left[\begin{array}{cccc}
\bar{e}_{1} & 1 & 0 & 0 \\
2 \bar{e}_{2} & \bar{e}_{1} & \ddots & 0 \\
\vdots & \vdots & \ddots & 1 \\
q \cdot \bar{e}_{q} & \bar{e}_{q-1} & \cdots & \bar{e}_{1}
\end{array}\right] \quad \forall q \in \mathbb{N} \text {, where } \bar{e}_{q} \equiv 0, \forall q>n \\
\bar{e}_{q}=\frac{1}{q !} \operatorname{det}\left[\begin{array}{cccc}
\bar{p}_{1} & 1 & 0 & 0 \\
\bar{p}_{2} & \bar{p}_{1} & \ddots & 0 \\
\vdots & \vdots & \ddots & q-1 \\
\bar{p}_{q} & \bar{p}_{q-1} & \cdots & \bar{p}_{1}
\end{array}\right] \quad \forall q \in \mathbb{N} .
\end{gathered}
$$

When $q>n$, formula (4.4) yields zero for $\bar{e}_{q}$. Formulae (4.1) through (4.4) immediately imply the equalities and inclusions $\mathbb{Q}\langle e\rangle_{m}=\mathbb{Q}\left\langle p_{1}, \ldots, p_{N}\right\rangle_{m}, \mathbb{Q}\langle e\rangle=\mathbb{Q}\left\langle p_{1}, \ldots, p_{N}\right\rangle$, $\mathbb{Z}\left\{p_{1}, \ldots, p_{N}\right\}_{m} \subset \mathbb{Z}\{e\}_{m} \subset \mathbb{Q}\{e\}_{m}=\mathbb{Q}\left\{p_{1}, \ldots, p_{N}\right\}_{m}, \mathbb{Z}\left\{p_{1}, \ldots, p_{N}\right\} \subset \mathbb{Z}\{e\} \subset \mathbb{Q}\{e\}=$ $\mathbb{Q}\left\{p_{1}, \ldots, p_{N}\right\}$ and the same with $\bar{e}$ in place of $e$ and $\bar{p}_{1}, \ldots, \bar{p}_{n}$ in place of $p_{1}, \ldots, p_{N}$.

For the proof of Theorem 9.1, we wish to find an element $U \in \mathbb{Z}[e]$, preferably of lowest possible weight when weight is defined, such that $U \cdot \bar{P} \in \mathbb{Z}[e, t]$. Because it is so easy to do so, we will prove something a little stronger. Let $S_{n}$ denote the full symmetric group on the $n$ roots of $\bar{P}$. Let $A_{n}$ denote the alternating subgroup consisting of all even permutations in $S_{n}$. We say that a polynomial $\eta \in \mathbb{Z}\left[z_{1}, \ldots, z_{n}\right]$ is antisymmetric in the roots if $g(\eta)=\eta$, for every $g \in A_{n}$, and $h(\eta)=-\eta$, for every $h \in S_{n}, h \notin A_{n}$. Let $\delta$ denote the partition $\delta \equiv(n-1, n-2, \ldots, 1,0)$. For any partition $\mu=\left(\mu_{1}, \ldots, \mu_{n}\right)$ of length $\ell(\mu) \leq n$, define the antisymmetric polynomial $a_{\mu} \equiv \sum_{g \in A_{n}} \prod_{i=1}^{n} z_{g(i)}^{\mu_{i}}-\sum_{g \in S_{n}, g \notin A_{n}} \prod_{i=1}^{n} z_{g(i)}^{\mu_{i}}$. Then $\mu$ may be written as a determinant, $a_{\mu}=\operatorname{det}\left[z_{i}^{\mu_{j}}\right]_{i, j \in[n]}$. Then the Vandermonde determinant form of the discriminant of $\bar{P}$ may be expressed as $\Delta=a_{\delta}^{2}$.

DeFinition 4.2. Define the Schur function indexed by the partition $\lambda$ to be $\bar{s}_{\lambda} \equiv$ $a_{\lambda+\delta} \div a_{\delta}$. The Schur function $\bar{s}_{\lambda}$ is a homogeneous symmetric polynomial in $\mathbb{Z}\left[z_{1}, \ldots, z_{n}\right]$ whose weight $w\left(\bar{s}_{\lambda}\right)$ equals $|\lambda|$ by Definition 5.1 . The bar reminds us that $\bar{S}_{\lambda}$ is symmetric in the roots of $\bar{P}$, not counting multiplicities.

Note that if any two parts of $\mu$ are equal, then $a_{\mu}=0$, which is the only element both symmetric and antisymmetric. Hence, we may assume $\mu$ has distinct parts and write it as $\mu=\lambda+\delta$, where $\lambda$ is the partition whose parts are defined by $\lambda_{i} \equiv \mu_{i}-n+i$.

Lemma 4.3. Define $U \equiv\left(\prod_{j=1}^{n} \pi_{j}\right) \cdot \Delta$. Let e and $\bar{e}$ be given by Definitions 2.5 and 2.6. Then $U \cdot \mathbb{Z}[\bar{e}] \subset \mathbb{Z}[e]$ and $U \in \mathbb{Z}[e]$. 
Proof. We have

$$
\begin{aligned}
U \cdot \bar{s}_{\lambda} & =\left(\prod_{j=1}^{n} \pi_{j} \cdot \Delta\right) \cdot \frac{a_{\lambda+\delta}}{a_{\delta}}=\left(\prod_{j=1}^{n} \pi_{j} \cdot a_{\delta}\right) \cdot a_{\lambda+\delta} \\
& =\operatorname{det}\left[\pi_{j} \cdot z_{j}^{i}\right]_{i \times j} \cdot \operatorname{det}\left[z_{j}^{\lambda_{k}+n-k}\right]_{j \times k} \\
& =\operatorname{det}\left[\sum_{j=1}^{n} \pi_{j} \cdot z_{j}^{i+\lambda_{k}+n-k}\right]_{i \times k} \\
& =\operatorname{det}\left[p_{i+\lambda_{k}+n-k}\right]_{i \times k} \in \mathbb{Z}[e] .
\end{aligned}
$$

By [14, formula (3.3), page 41], any $h \in \mathbb{Z}[\bar{e}]$ can be written as a finite sum of Schur functions over $\mathbb{Z}, h=\sum_{\lambda} b_{\lambda} \cdot \bar{s}_{\lambda}$ with $b_{\lambda} \in \mathbb{Z}$. So, $U \cdot h=\sum_{\lambda} b_{\lambda} \cdot\left(U \cdot \bar{s}_{\lambda}\right) \in \mathbb{Z}[e]$. This proves the first assertion.

If we let $\lambda=(0)$, we get $U=U \cdot 1=U \cdot \bar{s}_{(0)} \in \mathbb{Z}[e]$. This proves the second assertion.

Corollary 4.4. Let $e$ and $\bar{P}$ be given by Definitions 2.5 and 2.6. Then $U \cdot \bar{P} \in \mathbb{Z}[e, t]$.

Since the $q$ th powersum $\sum_{j=1}^{n} \pi_{j} \cdot z_{j}^{q}$ is not fixed under action by $S_{n}$ for arbitrary multiplicities $\pi_{j}$, we cannot say anything about the action of $S_{n}$ on the ring $\mathbb{Q}\left[p_{1}, \ldots, p_{N}\right]=$ $\mathbb{Q}[e]$. So we cannot use $S_{n}$ directly, nor the Galois group of $P$, for the final symmetrization of the resolvent of $\bar{P}$ to a resolvent of $P$ in Theorems 8.3 and 9.1. Instead we will apply Lemma 4.5 .

LEMMA 4.5. Let $o \in[n]$. Let $\eta$ be a linear combination of antisymmetric polynomials in $\mathbb{Z}\left[z_{1}, \ldots, z_{n}\right]$ over the ring $\mathbb{Z}\{e\}_{o}[\alpha]$. Then $U \cdot(\eta \div \sqrt{\Delta}) \in \mathbb{Z}\{e\}_{o}[\alpha]$.

Proof. By [14, page 40] we may write $\eta=\sum_{\mu} h_{\mu} \cdot a_{\mu}$ with $h_{\mu} \in \mathbb{Z}\{e\}_{o}[\alpha]$. Thus $U \cdot(\eta / \sqrt{\Delta})=\sum_{\mu} h_{\mu} \cdot\left(U \cdot\left(a_{\mu} / \sqrt{\Delta}\right)\right)=\sum_{\mu} h_{\mu} \cdot\left(U \cdot \bar{s}_{\mu-\delta}\right) \in \mathbb{Z}\{e\}_{o}[\alpha]$ since $U \cdot \bar{s}_{\mu-\delta} \in \mathbb{Z}[\bar{e}]$ by Lemma 4.3 .

\section{Weight of rational functions}

DEFINITION 5.1. Define the weight of a monomial $h=s \cdot \prod_{m \geq 0} \prod_{j=1}^{n}\left(D^{m} z_{j}\right)^{v_{j, m}} \cdot \alpha^{i}$, with $s \in \mathbb{Z}^{\#}$ in the ring $\mathbb{Z}\left\{z_{1}, \ldots, z_{n}\right\}[\alpha]$, by $w(h) \equiv \sum_{m \geq 0} \sum_{j=1}^{n} v_{j, m}$.

Thus weight is defined so that $w\left(z_{l}\right)=1$, for all $l \in[n], w(h)=0$, for all $h \in$ $(\mathbb{Z}[\alpha])^{\#}$, derivation leaves weight unchanged, and the weight of a polynomial $h \in$ $\mathbb{Z}\left\{z_{1}, \ldots, z_{n}\right\}[\alpha]$ is defined such that each of the coefficients in $\mathbb{Z}\left\{z_{1}, \ldots, z_{n}\right\}$ of the separate powers of $\alpha$ has the same weight.

DEFINITION 5.2. The weight of a monomial $\hat{h}=s \cdot \prod_{m \geq 0} \prod_{l=1}^{q}\left(D^{m} p_{\iota}\right)^{v_{l, m}} \cdot \alpha^{i}$, with $s \in \mathbb{Z}^{\#}$ in the ring $\mathbb{Z}\left\{p_{1}, \ldots, p_{q}\right\}[\alpha]$, is defined by $w(\hat{h}) \equiv \sum_{m \geq 0} \sum_{\iota=1}^{q} \iota \cdot v_{\iota, m}$.

Weight is well defined if any finite linear relation of these monomials can be decomposed as the sum of homogeneous finite linear relations of monomials. This means that 
if any monomial can be expressed as a linear combination over $\mathbb{Z}^{\#}$ of other monomials, then that monomial can be expressed as a linear combination over $\mathbb{Z}^{\#}$ of monomials of the same weight.

Let $h$ be a sum of monomials in $\mathbb{Z}\left\{z_{1}, \ldots, z_{n}\right\}[\alpha]$ all of the same weight. Then we call $h$ a homogeneous polynomial of that weight. The discriminant $\Delta$ of $\bar{P}$, for instance, is a homogeneous polynomial in $\mathbb{Z}[\bar{e}]$ of weight $n^{2}-n$. Clearly any homogeneous polynomial in $\mathbb{Z}\left\{p_{1}, \ldots, p_{q}\right\}[\alpha]$ is a homogeneous polynomial in $\mathbb{Z}\left\{z_{1}, \ldots, z_{n}\right\}[\alpha]$.

A ratio of two homogeneous polynomials, which need not have the same weight, is called a homogeneous rational function. Let $h, b \in \mathbb{Z}\left\{z_{1}, \ldots, z_{n}\right\}[\alpha]$ be homogeneous polynomials of weights $w(h)$ and $w(b)$, respectively. Then the weight of the rational function $h / b \in \mathbb{Q}\left\langle z_{1}, \ldots, z_{n}\right\rangle(\alpha)$ is defined to be $w(h)-w(b)$. It follows that if $h$ and $b$ have the same weight, then either $h-b$ is a nonzero homogeneous rational function of weight $w(h)=w(b)$, or $h-b=0$. The sum or difference of two homogeneous rational functions is not a homogeneous rational function if the weights of the summands are different. We define $w(0) \equiv \infty$ so that Definitions 5.1 and 5.2 are consistent with the property $w(0)=w(0 \cdot h)=w(0)+w(h)$, for all $h \in \mathbb{Q}\left\langle z_{1}, \ldots, z_{n}\right\rangle(\alpha)$.

DEFINITION 5.3. Call the distinct roots of $P$ differentially independent over constants if no finite sum of distinct nonzero monomials in $\mathbb{Z}\left\{z_{1}, \ldots, z_{n}\right\}$ equals 0 . Then call $P$ a differentially transcendental polynomial. Therefore define a differentially transcendental polynomial as one whose distinct roots satisfy the following: the field $\mathbb{Q}\left\langle z_{1}, \ldots\right.$, $\left.z_{n}\right\rangle_{m}$ has transcendence degree $n \cdot(m+1)$ over $\mathbb{Q}$ for every $m \in \mathbb{N}_{0}$.

\section{Definition and existence of resolvents}

DEFINITION 6.1. Define a nonzero homogeneous linear ordinary differential equation of finite order $\mathfrak{R} y \equiv \sum_{m \in \mathbb{N}_{0}} \sum_{i \in \mathbb{N}_{0}} r_{i, m} \cdot \alpha^{i} \cdot D^{m} y=0$ to be an $\alpha$-resolvent of $P$ if each $\alpha$-power $y \in\left\{y_{i}\right\}_{i=1}^{n}$ of the roots of $P$ satisfies this equation, if $\mathfrak{R}$ is polynomial in $\alpha$, and if each $r_{i, m}$ lies in the differential coefficient field $\mathbb{Q}\langle e\rangle$ of $P$.

DEFINITION 6.2. Call $r_{i, m}$ the $(i, m)$ th coefficient-function of $\mathfrak{R}$.

DEFINITION 6.3. Call the coefficient $R_{m} \equiv \sum_{i \in \mathbb{N}_{0}} r_{i, m} \cdot \alpha^{i}$ of $D^{m} y$ in $\mathfrak{R}$ the $m$ th term of $\mathfrak{R}$.

DEFINITION 6.4. Call $\mathfrak{R}$ homogeneous of weight $w$ if every $r_{i, m}$ has weight $w$ in the field $\mathbb{Q}\langle e\rangle$ whenever the weight of elements in $\mathbb{Q}\langle e\rangle$ is well defined.

DEFINITION 6.5. If the indeterminate power $\alpha$ is replaced with an integer power $q$, then call $\mathfrak{R}$ a $q$-resolvent. Technically, $\mathfrak{R}$ could be called a resolvent if the terms $R_{m}$ lie in $\mathbb{Q}\langle e\rangle(\alpha)$. However, for purposes of computing $\mathfrak{R}$ with the powersum formula [21], define resolvents as being polynomials in $\alpha$.

DEFINITION 6.6. When the coefficient-functions $r_{i, m}$ lie in the differential coefficient ring $\mathbb{Z}\{e\}$, call the resolvent integral.

Since the differential coefficient field of $\bar{P}$ lies in the differential coefficient field of $P$ by Lemma 2.8, and $P$ and $\bar{P}$ have the same roots modulo multiplicity, an $\alpha$-resolvent 
of $\bar{P}$ will be an $\alpha$-resolvent for $P$. However, an integral $\alpha$-resolvent of $\bar{P}$ will not be an integral $\alpha$-resolvent of $P$.

Clearly, linear combinations of the derivatives of resolvents over the ring $\mathbb{Q}\langle e\rangle[\alpha]$ are resolvents. We prefer that resolvents satisfy the following three conditions.

ConDITION 6.7. Resolvents should be of minimal order $o$.

Condition 6.8. Resolvents should have the fewest possible number of nonzero coefficient-functions.

Condition 6.9. Resolvents should be integral and have minimal weight.

A slight modification of the basic theory of homogeneous linear ordinary differential equations in [17, Theorem A2] guarantees the existence of resolvents, and not just linear ordinary differential equations whose terms lie in an arbitrary field, of $o$ th order, where $o$ is given by Definition 2.10. We will see that Condition 6.7 implies that the terms of integral $o$ th-order $\alpha$-resolvents lie in the smallest possible ring $\mathbb{Z}\{e\}_{o}[\alpha]$. The author asserts in [20, Theorems 18 and 19] that if no root is a constant multiple of another, then $o=n$ for indeterminate $\alpha$ and all but finitely many integers $\alpha=q$.

Among those resolvents which satisfy Condition 6.7, we will find those which satisfy Condition 6.8 in Theorem 8.3 and prove their uniqueness up to $\mathbb{Q}\langle e\rangle$-multiples in Theorem 10.1.

We may multiply a nonintegral resolvent which satisfies Conditions 6.7 and 6.8 by an appropriate factor in $\mathbb{Q}\langle e\rangle$ to obtain an integral resolvent. We may also multiply an integral resolvent by an appropriate factor in $\mathbb{Q}\langle e\rangle$ to obtain a possibly nonintegral resolvent but of zero weight, so as to make one of the coefficient-functions equal to 1 . Hence, minimizing the weight of resolvents in Condition 6.9 makes sense only for integral resolvents.

THEOREM 6.10. Define $\Omega \equiv o \cdot(o-1) / 2+1$. There exists an $\alpha$-resolvent of $P$ of the form $\sum_{m=0}^{o} \sum_{i=0}^{\Omega-m} \theta_{i, m} \cdot \alpha^{i} \cdot D^{m} y_{j}=0$ for some nonzero $\theta_{i, m} \in \mathbb{Z}\{e\}_{o}$ except $\theta_{0,0}=0$.

REMARK 6.11. Theorem 6.10 was originally proved as [18, Theorem 37, page 67]. It has been published in [17, Theorem A.2].

THEOREM 6.12. If $\Re$ is a resolvent of $P$, then $\Re$ may be expressed as a sum of homogeneous resolvents.

REMARK 6.13. Theorem 6.12 was originally [18, Theorem 3, page 25].

Therefore, Theorems 6.10 and 6.12 imply the following theorem.

THEOREM 6.14. There exists an integral $\alpha$-resolvent of $P$ of the form $\sum_{m=0}^{o} \sum_{i=0}^{\Omega-m} \theta_{i, m}$. $\alpha^{i} \cdot D^{m} y=0$ for some nonzero $\theta_{i, m} \in \mathbb{Z}\{e\}_{o}$ of minimal weight except $\theta_{0,0}=0$.

DEFINITION 6.15. Call the resolvent in Theorem 6.14 the Cohnian of P. Hence, the Cohnian is the $\alpha$ - or $q$-resolvent which satisfies Conditions $6.7,6.8$, and 6.9 in that order. Hereafter, the symbol $\theta_{i, m}$ will be used to represent the coefficient-functions of the Cohnian of $P$. 
7. Bell polynomials. For this section, we follow the notation of [14, page 30].

DeFINITION 7.1. The partial Bell polynomial $B_{m, k}\left\{z_{l}\right\} \in \mathbb{Z}\left\{z_{l}\right\}_{m}$ of the derivatives of a single root $z_{l}$ of $P$ is defined for each $m, k \in \mathbb{N}_{0}$ to be the coefficient of $z_{l}^{-k}$ in the expansion of $\left(D^{m} y_{l}\right) / y_{l}$ as a polynomial in $\alpha$.

Let $m \in \mathbb{N}$. Then $D^{m} y_{l}=y_{l} \cdot \sum_{k=1}^{m} B_{m, k}\left\{z_{l}\right\} \cdot(\alpha)_{k} \cdot z_{l}^{-k}$ with $(\alpha)_{k}$ given by Definition 2.3. Extend the definition of $B_{m, k}\left\{z_{l}\right\}$ to $m=0$ and $k=0$ to be consistent with $y_{l}=$ $D^{0} y_{l}$. So

$$
D^{m} y_{l}=y_{l} \cdot \sum_{k=0}^{m} B_{m, k}\left\{z_{l}\right\} \cdot(\alpha)_{k} \cdot z_{l}^{-k} .
$$

One immediately sees that $B_{m, 0}\left\{z_{l}\right\}=0$ for each $m \in \mathbb{N}, B_{0, k}\left\{z_{l}\right\}=0$ for each $k \in \mathbb{N}$, $B_{0,0}\left\{z_{l}\right\}=1$, and $B_{m, k}\left\{z_{l}\right\}=0$ for all $m, k \in \mathbb{N}_{0}$ with $k>m$. When the choice of root $z_{l}$ is understood, we will shorten $B_{m, k}\left\{z_{l}\right\}$ to $B_{m, k}$.

We have the following formula for $B_{m, k}$ on [14, page 31]:

$$
B_{m, k}=\sum_{\lambda} c_{\lambda} \cdot \prod_{j=1}^{m}\left(D^{j} z_{l}\right)^{r_{j}},
$$

where the sum is over all partitions $\lambda=\left(1^{r_{1}} \cdots n^{r_{n}}\right)$ of $m$ of length $k$, and $c_{\lambda} \equiv m ! \div$ $\prod_{j=1}^{n}\left(r_{j} ! \cdot(j !)^{r_{j}}\right) \in \mathbb{N}$. This implies $z^{m} \cdot\left(D^{m} y / y\right) \in \mathbb{Z}\{z\}_{m}[\alpha]$.

Formula (7.2) allows us to make the following two assertions about $B_{m, k}$. If the distinct roots are differentially independent over constants, then any one root $z_{l}$ and its derivatives are algebraically independent over constants. Therefore, monomials in the formula for $B_{m, k}$ indexed by distinct partitions $\lambda \neq \lambda^{\prime}$ cannot cancel. Therefore we have the following.

ASSERTION 7.2. If we can prove the existence of a certain partition $\lambda$ of $m$ of length $k$, then we have proven that a certain monomial exists in the expansion of $D^{m} y$ with nonzero integer coefficient $c_{\lambda}$.

The weight of $B_{m, k}$, by Definition 5.1, equals $\sum_{j=1}^{m} r_{j}=k$, the length of the partitions $\lambda$ appearing in (7.2). Since $k \in[m]$ implies $(m-k) \in[m-1]_{0}$ and $\sum_{j=2}^{m}(j-1) \cdot r_{j}=m-k$, we can find a partition $\lambda$ of $m$ of length $k$ given by $r_{1}=k-1$ and $r_{m-k+1}=1$ if $k<m$. Therefore we have the following.

ASSERTION 7.3. The degree of $D z_{l}$ in $B_{m, k}\left\{z_{l}\right\}$ equals $k-1$ and $B_{m, k}\left\{z_{l}\right\} \neq 0$ for each $k \in[m-1]$. For $k=m$, by Assertion 3.5, there exists only one partition of $m$ of length 1 , namely, the partition $\lambda=\left(m^{1}\right)=(m)$. For this partition of $m, c_{\lambda}=m ! \div\left((r !)(1 !)^{r}\right)=$ $m ! \div\left((m !)(1)^{m}\right)=1$. Therefore we have the following.

ASSERTION 7.4. For each $m \in \mathbb{N}_{0}$, we have $B_{m, m}=\left(D z_{l}\right)^{m} \neq 0$.

8. Form of resolvents of minimal order. The following quantities play a critical role in the form of an $\alpha$-resolvent of order $\boldsymbol{o}$.

DEFINITION 8.1. Define $\Omega \equiv\left(o^{2}-o+2\right) / 2$ as in Theorem 6.10. 
DeFinition 8.2. Define $\Psi \equiv n \cdot \Omega=n \cdot\left(o^{2}-o+2\right) / 2$.

THEOREM 8.3. Let $P$ be a differentially transcendental polynomial given by Definitions 2.5 and 5.3. Then there exists an integral $\alpha$-resolvent $\sum_{m=0}^{o} \sum_{i=0}^{\Omega-m} \theta_{i, m} \cdot \alpha^{i} \cdot D^{m} y_{j}=$ 0 of $P$ of minimal order which has all nonzero coefficient-functions $\theta_{i, m} \neq 0$ except $\theta_{00}=0$. The number $\Phi$ of nonzero coefficient-functions $\theta_{i, m}$ appearing in this equation is $\left(n^{3}-n^{2}+2 n+2\right) / 2$.

Proof. Set up the Wronskian form of a differential equation

$$
W_{\alpha}(t) \equiv\left|\begin{array}{cccc}
y_{1} & \cdots & y_{o} & t \\
\vdots & \ddots & \vdots & \vdots \\
D^{o} y_{1} & \cdots & D^{o} y_{o} & D^{o} t
\end{array}\right|
$$

where $W_{\alpha}(y)=0$ when $t$ is replaced with any $y$. We emphasize that the coefficients of $D^{m} t$ in $W_{\alpha}(t)$ do not lie in $\mathbb{Q}\langle e\rangle[\alpha]$ because this definition of $W_{\alpha}(t)$ is not symmetric in all of the roots of $P$ counting their multiplicities. Hence $W_{\alpha}$ is not an $\alpha$-resolvent for $P$. We will multiply $W_{\alpha}$ by the appropriate factor later to make it a resolvent of $P$.

DeFinition 8.4. Define $A_{m}$ to be the coefficient of $D^{m} t$ in $W_{\alpha}(t)$. Thus

$$
A_{m} \equiv(-1)^{m} \operatorname{det}\left[D^{m^{\prime}} y_{l}\right]_{m^{\prime} \neq m, m^{\prime} \in[o]_{0}, l \in[o]} .
$$

We need to prove the following two conditions hold.

Condition 8.5. The coefficient of $\alpha^{i}$ in $A_{m}$ is not zero for $o-1+\delta_{m, 0} \leq i \leq \Omega-m+$ $(o-1)$ for $m \in[o]_{0}$.

Condition 8.6. The coefficient of $\alpha^{i}$ in $A_{m}$ is zero for $i \in\left[o-2+\delta_{m, 0}\right]_{0}$ for $m \in$ $[o]_{0}$.

We first prove Condition 8.5 holds. For each $k \in \mathbb{N}$, by formula (7.1), we have $D^{m^{\prime}} y_{l}=$ $y_{l} \cdot z_{l}^{-m^{\prime}} \cdot \sum_{k=1}^{m^{\prime}} B_{m^{\prime}, k}\left\{z_{l}\right\} \cdot(\alpha)_{k} z_{l}^{m^{\prime}-k}$ for each $m^{\prime} \in[o]_{0}$. So

$$
D^{m^{\prime}} y_{l}=y_{l} \cdot z_{l}^{-m^{\prime}} \cdot\left((\alpha)_{m^{\prime}} \cdot\left(D z_{l}\right)^{m^{\prime}}+\sum_{k=1}^{m^{\prime}-1} B_{m, k}\left\{z_{l}\right\} \cdot(\alpha)_{k} z_{l}^{m^{\prime}-k}\right)
$$

where $B_{m^{\prime}, k}\left\{z_{l}\right\} \cdot(\alpha)_{k} z_{l}^{m^{\prime}-k} \in \mathbb{Z}[\alpha]\left\{z_{l}\right\}$ for $k \in\left[m^{\prime}-1\right]$ is a polynomial in $\alpha$ of degree $k$, which is less than or equal to $m^{\prime}-1$, and a polynomial in $D z_{l}$ of degree $k-1$ by Assertion 7.3. Note that $B_{m^{\prime}, k}\left\{z_{l}\right\} \cdot(\alpha)_{k} z_{l}^{m^{\prime}-k}$ is a homogeneous polynomial in $\mathbb{Z}\left\{z_{l}\right\}_{m^{\prime}}[\alpha]$ of weight $m^{\prime}$. Since no monomial in $B_{m^{\prime}, k}\left\{z_{l}\right\} \cdot(\alpha)_{k} z_{l}^{m^{\prime}-k}$ for $k<m^{\prime}$ contains $\left(D z_{l}\right)^{m^{\prime}}$, each monomial of $B_{m^{\prime}, k} \cdot(\alpha)_{k} z_{l}^{m^{\prime}-k}$ for $k<m^{\prime}$ must contain one of the derivatives of $z_{l}$ higher than one. Therefore, by (8.2) and (8.3), terms in $A_{m}$ involving only the zeroth and first derivatives of the roots come only from the term $\operatorname{det}\left[y_{l} \cdot z_{l}^{-m^{\prime}} \cdot(\alpha)_{m^{\prime}} \cdot\left(D z_{l}\right)^{m^{\prime}}\right]_{l \times m^{\prime}}$. Therefore, to prove Condition 8.5 holds for $A_{m}$, it is sufficient to prove Condition 8.5 holds for $\operatorname{det}\left[(\alpha)_{m^{\prime}} \cdot u_{l}^{m^{\prime}}\right]_{\substack{l \times m^{\prime} \\ l \in[o], m^{\prime} \in[o]}}$ where the $u_{l}$ are defined by $u_{l} \equiv D z_{l} / z_{l}$. 
We have

$$
\operatorname{det}\left[(\alpha)_{m^{\prime}} \cdot u_{l}^{m^{\prime}}\right]_{\substack{l \in[o], m^{\prime} \in[o]_{0}, m^{\prime} \neq m \\ l \times m^{\prime}}}=\prod_{\substack{i=0 \\ i \neq m}}^{o}(\alpha)_{i} \cdot \operatorname{det}\left[u_{l}^{m^{\prime}}\right]_{\substack{l \in[o], m^{\prime} \in[o]_{0}, m^{\prime} \neq m \\ m^{\prime}}}
$$

Since the $z_{l}$ are differentially independent over $\mathbb{Q}$, the $u_{l}$ are algebraically independent

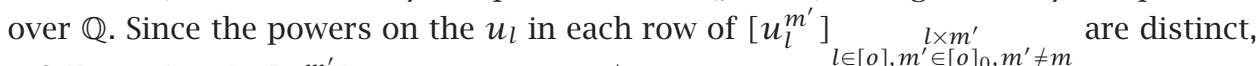
it follows that $\operatorname{det}\left[u_{l}^{m^{\prime}}\right]_{l \in[o], m^{\prime} \in[o]_{0}, m^{\prime} \neq m}^{\substack{l \times m^{\prime} \\ \text { ing }}} \neq 0$.

For each $m \in[o]_{0}$, we have $\prod_{\substack{m^{\prime} \neq 0 \\ m^{\prime}=0}}^{o}(\alpha)_{m^{\prime}}=\prod_{j=0}^{o-1}(\alpha-j)^{\eta_{j, m}}$, where $\eta_{j, m}=o-1-j$ for $j<m$ and $\eta_{j, m}=o-j$ for $j \geq m$. Therefore the degree of $\alpha$ in $\prod_{\substack{m^{\prime}=0 \\ m^{\prime} \neq m}}^{o}(\alpha)_{m^{\prime}}$ equals

$$
\begin{aligned}
\sum_{j=0}^{o-1} \eta_{j, m} & =\sum_{j<m}(o-1-j)+\sum_{j \geq m}(o-j) \\
& =\sum_{j=0}^{o-1}(o-j)-\#\left\{j \in[m-1]_{0}\right\}=\frac{o \cdot(o+1)}{2}-m .
\end{aligned}
$$

For each $m \in[o]$, we may factor $\alpha^{\eta_{0, m}}=\alpha^{o-1}$ from $\prod_{\substack{m^{\prime}=0 \\ m^{\prime} \neq m}}^{o}(\alpha)_{m^{\prime}}$. For $m=0$, we may factor $\alpha^{\eta_{0,0}}=\alpha^{o}$ from $\prod_{m^{\prime}=1}^{o}(\alpha)_{m^{\prime}}$. Note that $o \cdot(o+1) / 2-m-\left(o-1+\delta_{m, 0}\right)=\Omega-$ $m-\delta_{m, 0}$. So, $\prod_{j=0}^{o-1}(\alpha-j)^{\eta_{j, m}}=\alpha^{o-1+\delta_{m, 0}} \cdot \sum_{i=0}^{\Omega-m-\delta_{m, 0}}(-1)^{\Omega-m-\delta_{m, 0}-i} \rho_{i} \cdot \alpha^{i}$, where $\rho_{i}$ is the $\left(\Omega-m-\delta_{m, 0}-i\right)$ th elementary symmetric function of the positive integers, $j \in[o-1]$, where $j$ has multiplicity $\eta_{j, m}$. Therefore, $\rho_{i}>0$ for all $i \in\left[\Omega-m-\delta_{m, 0}\right]_{0}$, for all $m \in[o]_{0}$. Therefore, Condition 8.5 holds for $\operatorname{det}\left[(\alpha)_{m^{\prime}} \cdot u_{l}^{m^{\prime}}\right]_{l \in[o], m^{\prime} \in[o]_{0}, m^{\prime} \neq m}$. By earlier remarks Condition 8.5 holds for $A_{m}$.

We next prove Condition 8.6 holds. If $m^{\prime} \in[o]$, then the lowest power of $\alpha$ in $D^{m^{\prime}} y_{l}=$ $y_{l} \cdot \sum_{k=1}^{m^{\prime}} B_{m^{\prime}, k}\left\{z_{l}\right\} \cdot(\alpha)_{k} \cdot z_{l}^{-k}$ is 1 with coefficient $y_{l} \cdot \varsigma_{m^{\prime}, l}$, where

$$
\varsigma_{l, m^{\prime}} \equiv \sum_{k=1}^{m^{\prime}} B_{m^{\prime}, k}\left\{z_{l}\right\} \cdot z_{l}^{-k} \cdot(-1)^{k-1}(k-1) ! \in \mathbb{Z}\left\{z_{l}\right\}\left[z_{l}^{-1}\right]
$$

By Assertion 7.2, since the roots are differentially independent over constants, we need to find only one $k \in\left[m^{\prime}\right]$ and one partition of $m^{\prime}$ of length $k$ in formula (8.6) for $\varsigma_{l, m^{\prime}}$ to prove that $\varsigma_{l, m^{\prime}} \neq 0$. By Assertion 7.4, the partition $\lambda=\left(m^{\prime}\right)^{1}$ corresponding to $B_{m^{\prime}, m^{\prime}}\left\{z_{l}\right\} \neq 0$ will suffice. If $m^{\prime}=0$, then $D^{m^{\prime}} y_{l}=y_{l} \neq 0$. Therefore, we may write $D^{m^{\prime}} y_{l}=y_{l} \cdot\left(\alpha^{1-\delta_{m^{\prime}, 0}} \cdot \varsigma_{l, m^{\prime}}+\mathrm{O}_{l, m^{\prime}}\left(\alpha^{2}\right)\right)$, where $\mathrm{O}_{l, m^{\prime}}\left(\alpha^{2}\right)$ are terms in $\mathbb{Z}\left\{z_{l}\right\}\left[z_{l}^{-1}, \alpha\right]$ of degree in $\alpha$ strictly greater than 1 .

Therefore, for $m \in[o]_{0}$, we may pull out one power of $\alpha$ from the $m^{\prime}$ th column of $A_{m}=\operatorname{det}\left[D^{m^{\prime}} y_{l}\right]_{\substack{l \times m^{\prime} \\ m^{\prime} \neq m}}=\operatorname{det}\left[y_{l} \cdot\left(\alpha^{1-\delta_{m^{\prime}, 0}} \cdot \varsigma_{l, m^{\prime}}+\mathrm{O}_{l, m^{\prime}}\left(\alpha^{2}\right)\right)\right]_{\substack{l \times m^{\prime} \\ m^{\prime} \neq m}}$ except the 0th column, unless $m=0$, in which case we may pull out one power of $\alpha$ from each of 
the $o$ columns. Thus

$$
\begin{aligned}
A_{m} & =\left(\prod_{l=1}^{o} y_{l}\right) \cdot\left(\prod_{m^{\prime} \in[o]_{0}, m^{\prime} \neq m} \alpha^{1-\delta_{m^{\prime}, 0}}\right) \cdot\left(\operatorname{det}\left[\varsigma_{l, m^{\prime}}\right]_{\substack{l \times m^{\prime} \\
m^{\prime} \neq m}}+\mathrm{O}(\alpha)\right) \\
& =\left(\prod_{l=1}^{o} y_{l}\right) \cdot \alpha^{o-1+\delta_{m, 0}} \cdot\left(\operatorname{det}\left[\varsigma_{l, m^{\prime}}\right]_{\substack{l \times m^{\prime} \\
m^{\prime} \neq m}}+\mathrm{O}(\alpha)\right)
\end{aligned}
$$

where $\mathrm{O}(\alpha)$ are terms in $\mathbb{Z}\left\{z_{1}, \ldots, z_{o}\right\}\left[z_{1}^{-1}, \ldots, z_{o}^{-1}, \alpha\right]$ of degree in $\alpha$ strictly greater than zero.

We must now prove that $\operatorname{det}\left[\varsigma_{l, m^{\prime}}\right]_{\substack{l \times m^{\prime} \\ m^{\prime} \neq m}} \neq 0$ in (8.7). The highest-order derivative of $z_{l}$ which appears with nonzero coefficient in $\varsigma_{l, m^{\prime}}$ is $m^{\prime}$ when $k=1$ by formula (8.6) and Assertion 3.6. It is therefore easy to see that $\operatorname{det}\left[\varsigma_{l, m^{\prime}}\right]_{\substack{l \times m^{\prime} \\ m^{\prime} \neq m}}=\operatorname{det}\left[D^{m^{\prime}} z_{l} / z_{l}\right]_{\substack{l \times m^{\prime} \\ m^{\prime} \neq m}}+X$ in (8.7), where $X \in \mathbb{Z}\left\{z_{1}, \ldots, z_{o}\right\}\left[z_{1}^{-1}, \ldots, z_{o}^{-1}\right]$ has differential order strictly less than the differential order of $\operatorname{det}\left[D^{m^{\prime}} z_{l} / z_{l}\right]_{\substack{l \times m^{\prime} \\ m^{\prime} \neq m}}$. Therefore $\mathrm{X}$ cannot possibly cancel with $\operatorname{det}\left[D^{m^{\prime}} z_{l} / z_{l}\right]_{\substack{l \times m^{\prime} \\ m^{\prime} \neq m}}$ in (8.7) since the roots are differentially independent over constants. So, $\operatorname{det}\left[\varsigma_{l, m^{\prime}}\right]_{\substack{l \times m^{\prime} \\ m^{\prime} \neq m}} \neq 0$. Therefore, by (8.7), the lowest power of $\alpha$ with nonzero coefficient in $A_{m}$ is $o-1+\delta_{m, 0}$. Therefore the lowest power of $\alpha$ with nonzero coefficient in $A_{m} \div \alpha^{o-1}$ is $\delta_{m, 0}$.

We must now symmetrize $A_{m}$ with respect to all $N$ roots of $P$ including their multiplicities. First we specialize $o \rightarrow n$ (see Remarks 9.2) so that $\Omega \rightarrow n \cdot(n-1) / 2+1$, then multiply $A_{m}$ by enough powers of $\bar{e}_{n}$ and the square root of $\Delta$ until we get a differential equation whose coefficient-functions lie in $\mathbb{Z}\{e\}_{n}$. Specifically, if we define $R_{m}$ by (9.9) (see the proof of Theorem 9.1), then $\sum_{m=0}^{n} R_{m} \cdot D^{m} y=0$ will be an integral resolvent of $P$. After factoring out any other possible factors in $\mathbb{Z}\{e\}_{n}$, the resulting resolvent will be the Cohnian. The Cohnian will be a $\mathbb{Q}\langle\bar{e}, e\rangle(\sqrt{\Delta})$-multiple of $W_{\alpha}(t)$ and therefore have the same number of nonzero coefficient-functions for the same powers of $\alpha$ as $W_{\alpha}(t)$ does. This number equals $-1+\sum_{m=0}^{n}(\Omega+1-m)$, where the leading -1 accounts for the fact that $\theta_{0,0}=0$ and $\Omega+1-m$ equals the number of elements in $[\Omega-m]_{0}$, representing the distinct powers of $\alpha$ in the $m$ th term of the resolvent. Thus

$$
\begin{aligned}
\Phi & =-1+\sum_{m=0}^{n}(\Omega+1-m)=-1+(\Omega+1) \cdot(n+1)-\sum_{m=0}^{n} m \\
& =n \cdot \Omega+\Omega+n-\frac{n \cdot(n+1)}{2}=n \cdot \Omega+\Omega-\frac{n \cdot(n-1)}{2}=n \cdot \Omega+1=\Psi+1 .
\end{aligned}
$$

REMARK 8.7. Theorem 8.3 was originally [18, Theorem 40, page 71].

DEFINITION 8.8. Define $\Gamma \equiv\left\{(i, m) \ni i+m \in[\Omega], m \in[n]_{0}, i \geq 0\right\}$ to be the set of pairs $(i, m)$, which index the nonzero coefficients $\theta_{i, m}$ of $\alpha$ in the Cohnian of a differentially transcendental polynomial. By Theorem 8.3 and Definition 8.1, the size of $\Gamma$ equals $\Phi$. 


\section{Upper bound on the weight of the Cohnian}

THEOREM 9.1. Let the $\alpha$-powers $\left\{y_{i}\right\}_{i=1}^{n}$ of the $n$ distinct roots of $P$ given by Definition 2.5 be linearly independent over constants (so $o=n$ ). Then there exists an $\alpha$-resolvent $\sum_{m=0}^{n} R_{m} \cdot D^{m} y=0$ of $P$ with terms $R_{m} \in \mathbb{Z}\{e\}_{n}[\alpha]$ of weight $w\left(R_{m}\right)=n \cdot\left(4 n^{2}-\right.$ $5 n+3) / 2$ if $n=N$ and $w\left(R_{m}\right)=n \cdot\left(8 n^{2}-9 n+3\right) / 2$ if $n<N$.

Proof. If $n<N$, then, by Corollary 4.4, we have $U \equiv\left(\prod_{i=1}^{n} \pi_{i}\right) \cdot \Delta$ such that $U \in$ $\mathbb{Z}[e], U \cdot \bar{P}(t) \in \mathbb{Z}[e, t]$, and $w(U)=n^{2}-n$. If $n=N$, then we know $\bar{P}(t)=P(t)$. We may combine these two cases as $U^{1-\delta_{n, N}} \cdot \bar{P}(t) \in \mathbb{Z}[e, t]$, where $w\left(U^{1-\delta_{n, N}}\right)=\left(n^{2}-n\right)$. $\left(1-\delta_{n, N}\right)$. Define $\bar{S}(t) \equiv \partial \bar{P} / \partial t$ to be the separant of $\bar{P}$. Then $U^{1-\delta_{n, N}} \cdot \bar{S}(t) \in \mathbb{Z}[e, t]$. Since $\bar{S}\left(z_{j}\right) \neq 0$, the formulae in [12, pages 203-204] can be used to find polynomials $T_{m}(t) \in \mathbb{Z}\{e\}_{m}[t]$ such that

$$
D^{m} z_{j}=\frac{T_{m}\left(z_{j}\right)}{\left(U^{1-\delta_{n, N}} \bar{S}\left(z_{j}\right)\right)^{2 m-1}} \quad \forall j \in[n],
$$

with weight $w\left(T_{m}\left(z_{j}\right)\right)=1+(2 m-1) \cdot(n-1) \cdot\left(n+1-\delta_{n, N}\right)$ since $w\left(\bar{S}\left(z_{j}\right)\right)=n-1$ and $w(U)=n \cdot(n-1)$. Then

$$
\begin{aligned}
D^{m} y_{j} & =D^{m} z_{j}^{\alpha}=y_{j} \cdot \sum_{k=0}^{m} B_{m, k}\left\{z_{j}\right\} \cdot(\alpha)_{k} \cdot z_{j}^{-k} \\
& =y_{j} \cdot \sum_{k=0}^{m} \sum_{\lambda} c_{\lambda} \cdot \prod_{l=0}^{m}\left(D^{l} z_{j}\right)^{r_{l}} \cdot(\alpha)_{k} \cdot z_{j}^{-k} \\
& =y_{j} \cdot \sum_{k=0}^{m} \sum_{\lambda} c_{\lambda} \cdot \prod_{l=0}^{m}\left(\frac{T_{l}\left(z_{j}\right)}{\left(U^{1-\delta_{n, N}} \cdot \bar{S}\left(z_{j}\right)\right)^{2 l-1}}\right)^{r_{l}} \cdot(\alpha)_{k} \cdot z_{j}^{-k} \\
& =y_{j} \cdot \sum_{k=0}^{m} \frac{1}{\left(U^{1-\delta_{n, N}} \cdot \bar{S}\left(z_{j}\right)\right)^{\sum_{l=1}^{m}(2 l-1) \cdot r_{l}}} \sum_{\lambda} c_{\lambda} \cdot \prod_{l=0}^{m}\left(T_{l}\left(z_{j}\right)\right)^{r_{l}} \cdot(\alpha)_{k} \cdot z_{j}^{-k} \\
& =y_{j} \cdot \sum_{k=0}^{m} \frac{1}{\left(U^{1-\delta_{n, N}} \cdot \bar{S}\left(z_{j}\right)\right)^{2 m-k}} \sum_{\lambda} c_{\lambda} \cdot \prod_{l=0}^{m}\left(T_{l}\left(z_{j}\right)\right)^{r_{l}} \cdot(\alpha)_{k} \cdot z_{j}^{-k},
\end{aligned}
$$

where $\lambda=\left(1^{r_{1}} \cdots n^{r_{n}}\right), \sum_{l=1}^{m} r_{l}=k$, and $\sum_{l=1}^{m} l \cdot r_{l}=m$.

We defined $A_{m}$ in Definition 8.4 and gave a determinantal formula for it in (8.2). Combining formula (8.2) with formula (9.2) yields

$$
A_{m}=\operatorname{det}\left[y_{j} \cdot \sum_{k=0}^{m^{\prime}} \frac{1}{\left(U^{1-\delta_{n, N}} \cdot \bar{S}\left(z_{j}\right)\right)^{2 m^{\prime}-k}} \sum_{\lambda} c_{\lambda} \cdot \prod_{l=0}^{m^{\prime}}\left(T_{l}\left(z_{j}\right)\right)^{r_{l}} \cdot(\alpha)_{k} \cdot z_{j}^{-k}\right]_{\substack{j \times m^{\prime} \\ m^{\prime} \neq m, m^{\prime} \in[o]_{0}, j \in[o]}}
$$

The greatest negative power of $U^{1-\delta_{n, N}} \cdot \bar{S}\left(z_{j}\right)$ appearing in the $j$ th column of the determinantal formula (9.3) for $A_{m}$ is $2 o-1$ and occurs when $m^{\prime}=o$ and $k=0$ if $m \neq o$, and is $2 o-3$ and occurs when $m^{\prime}=o-1$ and $k=0$ if $m=o$. The greatest 
negative power of $z_{j}$ appearing in the $j$ th column of the determinantal formula (9.3) for $A_{m}$ is $o$ and occurs when $k=m^{\prime}=o$ if $m \neq o$, and is $o-1$ and occurs when $k=m^{\prime}=o-1$ if $m=o$. Therefore we will multiply formula (9.3) for $A_{m}$ by

$$
U^{(2 o-1) \cdot\left(1-\delta_{n, N}\right)} \cdot\left(\prod_{j=1}^{o} z_{j}^{o}\right) \cdot\left(\prod_{j=1}^{o}\left(\bar{S}\left(z_{j}\right)\right)^{2 o-1}\right) \div\left(\prod_{j=1}^{o} z_{j}^{\alpha}\right)
$$

so that the product will lie in the ring $\mathbb{Z}\left\{z_{1}, \ldots, z_{n}\right\}[\alpha]$.

So we define

$$
\begin{aligned}
H_{m} & \equiv U^{(2 o-1) \cdot\left(1-\delta_{n, N}\right)} \cdot\left(\prod_{j=1}^{o} z_{j}^{o}\right) \cdot\left(\prod_{j=1}^{o}\left(\bar{S}\left(z_{j}\right)\right)^{2 o-1}\right) \cdot A_{m} \div\left(\prod_{j=1}^{o} z_{j}^{\alpha}\right) \\
& =\operatorname{det}\left[H_{m^{\prime}, j}\right]_{\substack{j \times m^{\prime} \\
m^{\prime} \neq m, m^{\prime} \in[o]_{0}, j \in[o]}},
\end{aligned}
$$

where we define

$$
H_{m^{\prime}, j} \equiv \sum_{k=0}^{m^{\prime}}\left(U^{1-\delta_{n, N}} \cdot \bar{S}\left(z_{j}\right)\right)^{2 o-1-2 m^{\prime}+k} \sum_{\lambda} c_{\lambda} \cdot \prod_{l=0}^{m^{\prime}}\left(T_{l}\left(z_{j}\right)\right)^{r_{l}} \cdot(\alpha)_{k} \cdot z_{j}^{o-k}
$$

It is important to note that $H_{m^{\prime}, j}$ belongs to $\mathbb{Z}\{e\}_{m^{\prime}}\left[\alpha, z_{j}\right]$.

Let $\varepsilon \equiv \prod_{k=1}^{n} \pi_{k}$ so that $U=\varepsilon \cdot \Delta$. See Remarks 9.2 to explain why we must now specialize $o \rightarrow n$. Then

$$
\begin{aligned}
H_{m} & =U^{(2 n-1)\left(1-\delta_{n, N}\right)} \cdot\left(\prod_{j=1}^{n} z_{j}^{n}\right) \cdot\left(\prod_{j=1}^{n}\left(\bar{S}\left(z_{j}\right)\right)^{2 n-1}\right) \cdot A_{m} \div\left(\prod_{j=1}^{n} z_{j}^{\alpha}\right) \\
& =\varepsilon^{(2 n-1) \cdot\left(1-\delta_{n, N}\right)} \Delta^{(2 n-1) \cdot\left(1-\delta_{n, N}\right)} \cdot \bar{e}_{n}^{n} \cdot \Delta^{2 n-1} \cdot A_{m} \div\left(\prod_{j=1}^{n} z_{j}^{\alpha}\right) \\
& =\varepsilon^{(2 n-1) \cdot\left(1-\delta_{n, N}\right)} \Delta^{(2 n-1)\left(2-\delta_{n, N}\right)} \cdot \bar{e}_{n}^{n} \cdot A_{m} \div\left(\prod_{j=1}^{n} z_{j}^{\alpha}\right) .
\end{aligned}
$$

The weight of $H_{m}$ equals the weight of $\Delta^{(2 n-1)\left(2-\delta_{n, N}\right)} \cdot \bar{e}_{n}^{n} \cdot A_{m} \div\left(\prod_{j=1}^{n} z_{j}^{\alpha}\right)$ which equals

$$
\begin{aligned}
w\left(H_{m}\right) & =(2 n-1) \cdot n \cdot(n-1) \cdot\left(2-\delta_{n, N}\right)+n^{2}+w\left(A_{m}\right)-\alpha \cdot n \\
& =4 n^{3}-5 n^{2}+2 n-\delta_{n, N} \cdot(2 n-1) \cdot n \cdot(n-1) .
\end{aligned}
$$

Then $H_{m}$, being a Vandermonde-type determinant by formula (9.2), becomes a linear combination over $\mathbb{Z}\{e\}_{n}[\alpha]$ of antisymmetric polynomials in $\mathbb{Z}\left[z_{1}, \ldots, z_{n}\right]$. If $n=N$, then we may symmetrize $H_{m}$ by dividing by $\sqrt{\Delta}$. If $n<N$, then we must apply Lemma 4.5 which guarantees that $H_{m} \cdot(U \div \sqrt{\Delta})$ lies in $\mathbb{Z}\{e\}_{n}[\alpha]$. We may combine these two cases 
by defining

$$
\begin{aligned}
R_{m} & \equiv(-1)^{m} \cdot H_{m} \cdot \frac{U^{1-\delta_{n, N}}}{\alpha^{n-1} \sqrt{\Delta}} \\
& =(-1)^{m} \cdot \varepsilon^{2 n \cdot\left(1-\delta_{n, N}\right)} \Delta^{4 n-1-2 n \cdot \delta_{n, N}} \cdot \bar{e}_{n}^{n} \cdot \frac{A_{m}}{\alpha^{n-1} \sqrt{\Delta}} \div\left(\prod_{j=1}^{n} z_{j}^{\alpha}\right),
\end{aligned}
$$

where Condition 8.6 (after specializing $o \rightarrow n$ ) guarantees that we may divide $A_{m}$ by $\alpha^{n-1}$ and still leave a polynomial in $\alpha$. We grouped the terms in (9.9) as we did in order to emphasize that $A_{m} \div\left(\alpha^{n-1} \sqrt{\Delta} \prod_{j=1}^{n} z_{j}^{\alpha}\right)$ belongs to $\mathbb{Z}\left\{z_{1}, \ldots, z_{n}\right\}\left[z_{1}^{-1}, \ldots, z_{n}^{-1}, \alpha\right]$ and is symmetrical in the roots not counting multiplicities. Thus $\sum_{m=0}^{n} R_{m} \cdot D^{m} y=0$ is an integral $\alpha$-resolvent of $P$ and

$$
\begin{aligned}
w\left(R_{m}\right)= & w\left(H_{m}\right)+\left(1-\delta_{n, N}\right) \cdot w(U)-\frac{w(\Delta)}{2} \\
= & 4 n^{3}-5 n^{2}+2 n-\delta_{n, N} \cdot(2 n-1) \cdot n \cdot(n-1) \\
& +\left(1-\delta_{n, N}\right) \cdot n \cdot(n-1)-\frac{n \cdot(n-1)}{2} \\
= & n \cdot\left(\frac{8 n^{2}-9 n+3}{2}\right)-2 n^{2}(n-1) \cdot \delta_{n, N} \\
= & n \cdot\left(\frac{8 n^{2}-9 n+3}{2}\right) \quad \text { if } n<N, \\
= & n \cdot\left(\frac{4 n^{2}-5 n+3}{2}\right) \quad \text { if } n=N .
\end{aligned}
$$

REMARKS 9.2. Theorem 9.1 was originally [18, Theorem 46, page 95]. At the point in the proof just before we specialized $o \rightarrow n$, we would normally apply each element $g$ of the Galois group $G$ of $P$ to $H_{m}$ and then add up the $g\left(H_{m}\right)$ over $G$ in order to make the coefficient-functions of the differential equation symmetrical with respect to the roots of $P$ including their multiplicities. However, there is no guarantee the result $\hat{H}_{m} \equiv \sum_{g \in G} g\left(H_{m}\right)$ will not be identically zero for each $m \in[o]_{0}$. We can only guarantee that if $\hat{H}_{o}=0$, then $\hat{H}_{m}=0$, for all $m \in[o]_{0}$, by the linear independence of $\left\{y_{i}\right\}_{i=1}^{o}$, since $\sum_{m=0}^{o} \hat{H}_{m} \cdot(-1)^{m} D^{m} y=0$.

The upper bound $\varphi(n) \equiv n \cdot\left(4 n^{2}-5 n+3\right) / 2$ in Theorem 9.1 is not sharp on the weight of the Cohnian for a quadratic and a cubic with distinct roots. The Cohnian of the quadratic, given in [17] and [18, page 131], has weight 7 , yet $\varphi(2)=9$. The Cohnian of the cubic has weight 24 , as demonstrated by indirect computer calculations in [18], yet $\varphi(3)=36$.

The proof of Theorem 9.1 suggests that exactly one power of the discriminant $\Delta$ of $P$ divides the leading term of the Cohnian in the ring $\mathbb{Z}\{e\}[\alpha]$ and none of the other terms. The discriminant in the leading term and not in the others has been observed in every resolvent of every polynomial the author has tested. This pattern seems to have been missed by Belardinelli in [1], Cayley in [2], and Cockle in [3, 4, 5, 6, 7]. It is consistent with the fact that the singularities of the resolvent occur at values of the implicit 
independent variable $x$ which make two or more of the roots of the polynomial equal. A purely algebraic proof that exactly one more power of $\Delta$ divides $H_{o}$ rather than $H_{m}$ for $m<o$ would require more than the Wronskian-type formulae used in the proof of Theorem 9.1.

10. Uniqueness of resolvents up to a multiple. For Theorem 10.1, we may assume $\Omega=n \cdot(n-1) / 2+1, \Psi=n \cdot \Omega$, and $\Phi=\Psi+1$.

THEOREM 10.1. Let $P$ be a differentially transcendental polynomial given by Definitions 2.5 and 5.3 with $n$ distinct roots. Let $\Re y \equiv \sum_{m=0}^{n} \sum_{i=0}^{\Omega-m} r_{i, m} \cdot \alpha^{i} \cdot D^{m} y=0$ be any $n$ th-order $\alpha$-resolvent of $P$ whose $m$ th term has degree in $\alpha$ less than or equal to $\Omega-m$. Then all $r_{i, m} \neq 0$ except $r_{0,0}=0$.

Proof. If we specialize the $\alpha$-resolvent $\mathfrak{R}$ to a $q$-resolvent by $\alpha \rightarrow q$, then, summing over the roots counting multiplicities, we get $\sum_{m=0}^{n} \sum_{i=0}^{\Omega-m} r_{i, m} \cdot q^{i} \cdot D^{m} p_{q}=0$ for

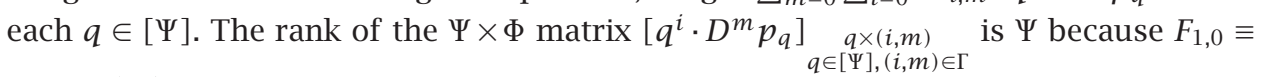
$(-1)^{\operatorname{sgn}(1,0)}\left[q^{i} \cdot D^{m} p_{q}\right] \underset{q \in[\Psi],(i, m) \neq(1,0)}{q \times(i, m)} \neq 0$ by [19, Theorem 4.1]. Also the $\Phi$ coefficientfunctions $\theta_{i, m}$ satisfy the same system of $\Psi$ linear equations $\sum_{m=0}^{n} \sum_{i=0}^{\Omega-m} \theta_{i, m} \cdot q^{i}$. $D^{m} p_{q}=0$ for each $q \in[\Psi]$. Therefore, $r_{i, m}=r_{1,0} \cdot\left(F_{i, m} \div F_{1,0}\right)=r_{1,0} \cdot\left(\theta_{i, m} \div \theta_{1,0}\right)$, for all $(i, m) \in \Gamma$, by Definition 8.8. By Theorem 8.3, $\left(\theta_{i, m} \div \theta_{1,0}\right) \neq 0$, for all $(i, m) \in \Gamma$. Thus, either $r_{i, m} \neq 0$, for all $(i, m) \in \Gamma$, or $r_{i, m}=0$, for all $(i, m) \in \Gamma$. But the latter case violates the definition of a resolvent. Therefore $r_{i, m} \neq 0$, for all $(i, m) \in \Gamma$.

REMARKS 10.2. Theorem 10.1 implies that any $n$ th-order $\alpha$-resolvent of $P$ with coefficient-functions indexed by $\Gamma$ is a $\mathbb{Q}\langle e\rangle$-multiple of the Cohnian. The author made this claim in [19, Theorem 4.1] by referring to [18, Theorem 40, page 71]. However, the author has not presented the argument in Theorem 10.1 in a published form. Therefore, Theorem 10.1 has been presented here to fill this gap. The reader may be interested to know why the proof of Theorem 10.1 had to use the powersums, implying the use of the powersum formula, instead of just using the system of $\Psi$ linear $q$-resolvents $\sum_{(i, m) \in \Gamma} r_{i, m} \cdot q^{i} \cdot D^{m} z_{j}^{q}=0$ for $j \in[n], q \in[\Omega]$. The reason is that $\operatorname{rank}\left[q^{i} \cdot D^{m} z_{j}^{q}\right] \underset{(i, m) \in \Gamma,(j, q) \in[n] \times[\Omega]}{(i, m) \times(j, q)}<\Psi$, and therefore this matrix could not be used to determine uniqueness of the coefficient-functions up to a common multiple.

11. Indicial equation of the trinomial resolvent. Cockle in [4] and Belardinelli in [1] used the Lagrange inversion formula

$$
z^{\alpha}=1+\frac{\alpha}{n} \sum_{k=1}^{\infty} \frac{(-1)^{k}}{k}\left(\begin{array}{c}
\frac{p \cdot k+\alpha}{n}-1 \\
k-1
\end{array}\right) \cdot x^{k}
$$

to compute a particular $\alpha$-resolvent of $P(t) \equiv t^{n}+x \cdot t^{p}-1$. Egorychev in [9] provides an easily accessible reference for the Lagrange inversion formula. We will now show how their resolvent also follows from the toric partial differential equations (11.4) of 
Mayr [15] and Sturmfels [23] and the Euler homogeneity partial differential equations (11.2) and (11.3), which are special cases of the $A$-hypergeometric partial differential equations of Gel'fand in [10, page 404].

DEFINITION 11.1. Let $\left(\begin{array}{c}m \\ j\end{array}\right)$ denote the binomial coefficient $m ! /((m-j) ! \cdot j !)$.

DEFinition 11.2. Define the shift operator $\tau$ as in [22, formula (3), page 2] to be $\tau f(t) \equiv f(t+1)-f(t)$ on any polynomial $f(t)$ of $t$.

DEFINITION 11.3. Similar to Definition 2.3, for any variable or operator $v$, any $m \in$ $\mathbb{N}$, and any $r \in \mathbb{Q}$, define $\langle v\rangle_{m, r} \equiv \prod_{k=1}^{m}(v+(k-1) \cdot r)$ as in [8]. Define $\langle v\rangle_{0, q} \equiv 1$.

DEFINITION 11.4. Define $\left.\tau^{m} f(t)\right|_{t \rightarrow 0}$ to be $f(t)$ operated on by the shift operator $\tau, m$ times, then evaluated at $t=0$. Define $\left.\tau^{0} f(t)\right|_{t \rightarrow 0} \equiv f(0)$.

DEFINITION 11.5. Given a set of $n+1$ indeterminates $\left\{a_{k}\right\}_{k=0}^{n}$ for each $k \in[n]_{0}$, the operator $\partial_{k}$ denotes the partial derivative $\partial / \partial a_{k}$ with respect to $a_{k}$ holding all other $a_{i \neq k}$ fixed.

The $\alpha$-power $z^{\alpha}$ of any root $z$ of the polynomial $\hat{P}(t) \equiv \sum_{i=0}^{n} a_{i} \cdot t^{i}$ satisfies the partial differential equations defined by (11.2), (11.3), and (11.4).

DEFINITION 11.6. The first Euler homogeneity partial differential equation is given by

$$
\sum_{k=0}^{n} a_{k} \cdot \partial_{k} z^{\alpha}=0
$$

It is easy to prove (11.2) by applying the operator $\sum_{k=0}^{n} a_{k} \cdot \partial_{k}$ to $\sum_{i=0}^{n} a_{i} \cdot z^{i}=0$.

DEFINITION 11.7. The second Euler homogeneity partial differential equation is given by

$$
\sum_{k=0}^{n} k \cdot a_{k} \cdot \partial_{k} z^{\alpha}+\alpha \cdot z^{\alpha}=0
$$

It is easy to prove (11.3) by applying $\sum_{k=0}^{n} k \cdot a_{k} \cdot \partial_{k}$ to $\sum_{i=0}^{n} a_{i} \cdot z^{i}=0$.

DEFINITION 11.8. The toric partial differential equation homogeneous in weight and total order of partial derivatives is given for each $p \in[n]_{0}$ by

$$
\partial_{p}^{n} z^{\alpha}=\partial_{n}^{p} \partial_{0}^{q} z^{\alpha}
$$

THEOREM 11.9. Let $P(t) \equiv t^{n}+x \cdot t^{p}-1$ be a trinomial with roots $\left\{z_{k}\right\}_{k=1}^{n}$. Let $q \equiv n-$ $p, \alpha \in \mathbb{Z}, D x \equiv 1$, and $y=z^{\alpha}$. Then Cockle's [6, formula (vi), page 186] and Belardinelli's [1, formula (8), page 467] $\alpha$-resolvent $D^{n} y=(-p)^{p} q^{q} / n^{n} \cdot\langle x \cdot D+\alpha / p\rangle_{p, n / p}\langle x \cdot D-$ $\alpha / q\rangle_{q, n / q} y$ of $P$ follows from (11.2), (11.3), and (11.4) by differential specialization. 
Proof. Let $a_{i} \rightarrow 0$ for $i \notin\{0, p, n\}$ and $a_{p} \rightarrow x$ in the polynomial $P(t) \equiv \sum_{i=0}^{n} a_{i} \cdot t^{i}$. Then (11.3) specializes to

$$
\left(p \cdot x \cdot \partial_{p}+n \cdot a_{n} \cdot \partial_{n}\right) y+\alpha \cdot y=0
$$

Therefore

$$
\left(n \cdot a_{n} \cdot \partial_{n}\right) y=\left(-p \cdot x \cdot \partial_{p}-\alpha\right) y
$$

Therefore

$$
\left(n \cdot a_{n} \cdot \partial_{n}\right)_{p, n} y=\left(-p \cdot x \cdot \partial_{p}-\alpha\right)_{p, n} y
$$

By the identity $(-v)_{m, r}=(-1)^{m}\langle v\rangle_{m, r}$ with $v=p \cdot x \cdot \partial_{p}+\alpha, m=p$, and $r=n$, we have

$$
\left(n \cdot a_{n} \cdot \partial_{n}\right)_{p, n} y=(-1)^{p}\left\langle p \cdot x \cdot \partial_{p}+\alpha\right\rangle_{p, n} y
$$

By the identity $(r \cdot v)_{m, r}=r^{m}\langle v\rangle_{m, 1}$ with $v=a_{n} \cdot \partial_{n}, m=p$, and $r=n$, we have

$$
n^{p} \cdot\left(a_{n} \cdot \partial_{n}\right)_{p, 1} y=(-1)^{p}\left\langle p \cdot x \cdot \partial_{p}+\alpha\right\rangle_{p, n} y
$$

By the differential identity $\left(a_{n} \cdot \partial_{n}\right)_{p, 1}=a_{n}^{p} \cdot \partial_{n}^{p}$, we have

$$
a_{n}^{p} \cdot \partial_{n}^{p} y=(-n)^{-p} \cdot\left\langle p \cdot x \cdot \partial_{p}+\alpha\right\rangle_{p, n} y
$$

Similarly, the identity (11.2) specializes to $\left(a_{0} \cdot \partial_{0}+x \cdot \partial_{p}+a_{n} \cdot \partial_{n}\right) y=0$. Therefore

$$
\left(n \cdot a_{0} \cdot \partial_{0}\right) y=\left(-n \cdot x \cdot \partial_{p}-n \cdot a_{n} \cdot \partial_{n}\right) y .
$$

Therefore

$$
\left(n \cdot a_{0} \cdot \partial_{0}\right)_{q, n} y=\left(-n \cdot x \cdot \partial_{p}-n \cdot a_{n} \cdot \partial_{n}\right)_{q, n} y
$$

By (11.5), we have

$$
\left(n \cdot a_{0} \cdot \partial_{0}\right)_{q, n} y=\left(-n \cdot x \cdot \partial_{p}+p \cdot x \cdot \partial_{p}+\alpha\right)_{q, n} y
$$

Therefore

$$
\left(n \cdot a_{0} \cdot \partial_{0}\right)_{q, n} y=\left(-q \cdot x \cdot \partial_{p}+\alpha\right)_{q, n} y
$$

By the identity $(-v)_{m, r}=(-1)^{m}\langle v\rangle_{m, r}$ with $v=q \cdot x \cdot \partial_{p}-\alpha, m=q$, and $r=n$, we have

$$
\left(n \cdot a_{0} \cdot \partial_{0}\right)_{q, n} y=(-1)^{q}\left\langle q \cdot x \cdot \partial_{p}-\alpha\right\rangle_{q, n} y .
$$

By the identity $(r \cdot v)_{m, r}=r^{m}\langle v\rangle_{m, 1}$ with $v=a_{0} \cdot \partial_{0}, m=q$, and $r=n$, we have

$$
n^{q} \cdot\left(a_{0} \cdot \partial_{0}\right)_{q, 1} y=(-1)^{q}\left\langle q \cdot x \cdot \partial_{p}-\alpha\right\rangle_{q, n} y
$$


By the differential identity $\left(a_{0} \cdot \partial_{0}\right)_{p, 1}=a_{0}^{q} \cdot \partial_{0}^{q}$, we have

$$
\left(a_{0}^{q} \cdot \partial_{0}^{q}\right) y=(-n)^{-q}\left\langle q \cdot x \cdot \partial_{p}-\alpha\right\rangle_{q, n} y
$$

We now choose the particular toric identity $\partial_{p}^{n} y=\partial_{n}^{p} \partial_{0}^{q} y$ in (11.4) and multiply it on the left by $a_{n}^{p} \cdot a_{0}^{q}$ to get the identity

$$
a_{n}^{p} \cdot a_{0}^{q} \cdot \partial_{p}^{n} y=a_{n}^{p} \cdot a_{0}^{q} \cdot \partial_{n}^{p} \partial_{0}^{q} y=\left(a_{n}^{p} \cdot \partial_{n}^{p}\right)\left(a_{0}^{q} \cdot \partial_{0}^{q}\right) y
$$

Note that $a_{0}^{q}$ and $\partial_{n}^{p}$ commute in (11.18) because the partial derivative $\partial_{n}$ has no effect on $a_{0}$. By (11.10) and (11.17), the identity (11.18) becomes

$$
a_{n}^{p} \cdot a_{0}^{q} \cdot \partial_{p}^{n} y=(-n)^{-p} \cdot(-n)^{-q}\left\langle p \cdot x \cdot \partial_{p}+\alpha\right\rangle_{p, n}\left\langle q \cdot x \cdot \partial_{p}-\alpha\right\rangle_{q, n} y .
$$

Since $\partial_{p}$ is the only partial derivation operator in (11.19), we may replace it with the ordinary derivation operator $D$. Specializing $a_{n} \rightarrow 1$ and $a_{0} \rightarrow-1$ in (11.19) gives us

$$
(-1)^{p} \cdot D^{n} y=n^{-n} \cdot\langle p \cdot x \cdot D+\alpha\rangle_{p, n}\langle q \cdot x \cdot D-\alpha\rangle_{q, n} y
$$

Dividing both sides by $(-1)^{p} \cdot p^{p} \cdot q^{q}$ yields the resolvent in the form given in [1]

$$
D^{n} y=\frac{(-p)^{p} q^{q}}{n^{n}} \cdot\left\langle x \cdot D+\frac{\alpha}{p}\right\rangle_{p, n / p}\left\langle x \cdot D-\frac{\alpha}{q}\right\rangle_{q, n / p} y .
$$

How do we know that there does not exist a resolvent for $z^{n}+x \cdot z^{p}-1=0$ of order less than $n$ ? Let $d \equiv \operatorname{gcd}(n, p)$ denote the greatest common divisor of $n$ and $p$. The discriminant $\Delta$ of this trinomial is given by [10, page 406] as

$$
\Delta(x)=(-1)^{(n+2) \cdot(n-1) / 2} \cdot\left(n^{n / d}-(-p)^{p / d} q^{q / d} x^{n / d}\right)^{d} .
$$

THEOREM 11.10. The indicial equation for Cockle's resolvent of the trinomial $z^{n}+$ $x \cdot z^{p}-1=0$ is $\mathrm{I}(\xi)=(\xi)_{n}$ which has roots $[n-1]_{0}$.

Proof. First we will express Cockle's resolvent in the form $\sum_{i=0}^{n} x^{i} \cdot C_{i}(x) \cdot D^{i} y=0$, where $C_{i}(x) \in \mathbb{Q}(x)$. To do this, we use Cockle's resolvent as expressed in the form (11.21). By the Gregory-Newton series [22, formula (44), page 9], we have

$$
\left\langle v+\frac{\alpha}{p}\right\rangle_{p, n / p}\left\langle v-\frac{\alpha}{q}\right\rangle_{q, n / q}=\sum_{i=0}^{n} c_{i, f} \cdot(v)_{i},
$$

where

$$
\begin{aligned}
f=f(v) & \equiv\left\langle v+\frac{\alpha}{p}\right\rangle_{p, n / q}\left\langle v-\frac{\alpha}{q}\right\rangle_{q, n / q}, \\
c_{i, f} & \left.\equiv \frac{1}{i !} \tau^{i} f(v)\right|_{v \rightarrow 0} \in \mathbb{Q}[\alpha] .
\end{aligned}
$$


Replacing $v$ with $x \cdot D$, using the fact that $(x \cdot D)_{i}=x^{i} \cdot D^{i}$, and multiplying (11.21) by $(-1)^{(n+2) \cdot(n-1) / 2} \cdot n^{n}$ gives us

$$
0=(-1)^{(n+2) \cdot(n-1) / 2} \cdot\left(n^{n} D^{n} y-(-p)^{p} q^{q} \sum_{i=0}^{n} c_{i, f} \cdot x^{i} \cdot D^{i} y\right)
$$

which has the leading term $\Delta_{1}(x) \equiv(-1)^{(n+2) \cdot(n-1) / 2} \cdot\left(n^{n}-(-p)^{p} q^{q} c_{n, f} \cdot x^{n}\right)$.

By [22, Example 1.41, page 25], it is easy to compute $c_{n, f}=\left.\tau^{n} f(v)\right|_{v \rightarrow 0} \div n !=n ! \div$ $n !=1$. Therefore, by (11.22), the leading term equals $\Delta(x)$ if $d$ were 1 . Hence we denote it by $\Delta_{1}(x)$. So $\Delta_{1}(x)=(-1)^{(n+2) \cdot(n-1) / 2} \cdot\left(n^{n}-(-p)^{p} q^{q} x^{n}\right)$. Therefore

$$
0=\Delta_{1}(x) \cdot D^{n} y-(-1)^{(n+2) \cdot(n-1) / 2} \cdot(-p)^{p} q^{q} \sum_{i=0}^{n-1} c_{i, f} \cdot x^{i} \cdot D^{i} y
$$

Multiplying (11.26) by $x^{n} \div \Delta_{1}(x)$ and defining

$$
C_{i}(x) \equiv-\frac{(-1)^{(n+2) \cdot(n-1) / 2} \cdot p^{p} q^{q} \cdot x^{n}}{\Delta_{1}(x)} \cdot c_{i, f}
$$

for $i \in[n-1]_{0}$ and $C_{n}(x) \equiv 1$, yields a resolvent of the form $\sum_{i=0}^{n} x^{i} \cdot C_{i}(x) \cdot D^{i} y=0$. These rational functions are clearly all analytic at $x=0$. According to [11, page 397], the indicial equation of this ordinary differential equation is $\mathrm{I}(\xi)=\sum_{i=0}^{n}(\xi)_{i} \cdot C_{i}(0)=(\xi)_{n}$ which has roots $[n-1]_{0}$.

12. Apparent singularities. We will now prove that linear relations of $\left\{z_{i}^{\alpha}\right\}_{i=1}^{n}$ for a trinomial hold only for a finite set of integers $\alpha$, and we will find this set.

Definition 12.1. Define $\sigma \equiv e^{2 \pi \cdot \sqrt{-1} /(n / d)}$.

DEFINITION 12.2. Define $\omega \equiv e^{2 \pi \cdot \sqrt{-1} / n}$.

Definition 12.3. Define $\omega_{l} \equiv \omega^{l}$ for each $l \in[n]$.

THEOREM 12.4. Let $n \geq 3, p \in[n-1]$, and $d \equiv \operatorname{gcd}(n, p)$. Then Cockle's $\alpha$-resolvent (11.26) $0=\Delta_{1}(x) \cdot D^{n} y-(-1)^{(n+2) \cdot(n-1) / 2} \cdot(-p)^{p} q^{q} \sum_{i=0}^{n-1} c_{i, f} \cdot x^{i} \cdot D^{i} y$ of $P(t) \equiv$ $t^{n}-x \cdot t^{p}-1$ has the set $\left\{z_{i}^{\alpha}\right\}_{i=1}^{n}$ as a fundamental system of $n$ solutions, that is, the $\left\{z_{i}^{\alpha}\right\}_{i=1}^{n}$ are linearly independent over $\mathbb{Q}$ if and only if $d=1$ and $\alpha \notin\{0\} \cup \bigcup_{m=2}^{n-1} \bigcup_{k=1}^{m-1}(k$. $n-m \cdot p)$. The Cockle resolvent has actual singularities at the points $x \in\left\{n \cdot(-p)^{-p / n}\right.$. $\left.q^{-q / n} \cdot \sigma^{j}\right\}_{j=1}^{j=n / d}$ and apparent singularities at the points $x \in\left\{n \cdot(-p)^{-p / n} \cdot q^{-q / n}\right.$. $\left.\omega^{j}\right\}_{j=1}^{j=n}$, where $j$ is not divisible by $d$.

Proof. By a standard result in the theory of ordinary differential equations, found in [11, page 119] for example, there exist $n$ solutions $\left\{u_{i}\right\}_{i=1}^{n}$ of (11.26) which are linearly independent over the subfield of constants $\mathbb{Q}$. The $\alpha$-powers of the roots $z^{\alpha}$ of $P(t) \equiv$ $t^{n}-x \cdot t^{p}-1$ are certain linear combinations of $\left\{u_{i}\right\}_{i=1}^{n}$ and hence may not be linearly independent over $\mathbb{Q}$. 
The discriminant $\Delta(x)$ has roots $x \in\left\{n \cdot(-p)^{-p / n} \cdot q^{-q / n} \cdot \sigma^{j}\right\}_{j=1}^{j=n / d}$ by inspection of (11.22). These are the actual singularities of the trinomial. The leading term $\Delta_{1}(x)$ of the resolvent (11.26) has singularities $x \in\left\{n \cdot(-p)^{-p / n} \cdot q^{-q / n} \cdot \omega^{j}\right\}_{j=1}^{j=n}$ by inspection of (11.22) with $d=1$. Pulling out those singularities which are actual singularities leaves the apparent singularities.

Let $W(x)$ denote the Wronskian of the Cockle resolvent of $P(t)$. We will now prove that $W(0) \neq 0$ under the conditions on $d$ and $\alpha$ stated in the theorem. This will be sufficient to prove that $W(x)$ is not identically zero for these $d$ and $\alpha$, and thus the theorem will be proved. Let $L_{\alpha}(x)$ be the series given by the Lagrange inversion formula (11.1). Then $y=L_{\alpha}(x)$ is a solution for $z^{\alpha}$, where $z^{n}-x \cdot z^{p}-1=0$. As Mellin noted in [16], $\omega_{l} \cdot L_{1}\left(\omega_{l}^{p} \cdot x\right)$ generates all the $n$ distinct roots of $z^{n}-x \cdot z^{p}-1=0$ as we let $l$ range over $[n]$. Since $L_{1}(0)=L_{\alpha}(0)=1$ for all $\alpha \in \mathbb{Z}$ by (11.1), it follows that $L_{1}(x)$ and $L_{\alpha}(x)$ represent different powers of the same root $z_{1}(x)$. Therefore, $L_{\alpha}(x)=\left(L_{1}(x)\right)^{\alpha}$. Therefore $z^{\alpha}=\left(\omega \cdot L_{1}\left(\omega^{p} x\right)\right)^{\alpha}=\omega^{\alpha} \cdot\left(L_{1}\left(\omega^{p} x\right)\right)^{\alpha}=\omega^{\alpha} \cdot L_{\alpha}\left(\omega^{p} x\right)$.

Suppress the subscript $l$ on $\omega_{l}$ in formula (12.1) and (12.2). For $m \in[n-1]$,

$$
\begin{aligned}
\left.\frac{d^{m} \omega^{\alpha} L_{\alpha}\left(\omega^{p} x\right)}{d x^{m}}\right|_{x \rightarrow 0} & =\omega^{\alpha+p \cdot m} \frac{\alpha}{n} m ! \cdot \frac{(-1)^{m}}{m}\left(\begin{array}{c}
\frac{p \cdot m+\alpha}{n}-1 \\
m-1
\end{array}\right) \\
& =\omega^{\alpha+p \cdot m} \frac{\alpha}{n} \cdot((m-1) !) \cdot \frac{((p \cdot m+\alpha-n) / n)_{m-1}}{(m-1) !} \\
& =\omega^{p \cdot m+\alpha} \frac{\alpha}{n}\left(\frac{p \cdot m+\alpha-n}{n}\right)_{m-1}
\end{aligned}
$$

For $m=0$, we have

$$
\left.\frac{d^{m} \omega^{\alpha} \cdot L_{\alpha}\left(\omega^{p} x\right)}{d x^{m}}\right|_{\substack{x \rightarrow 0 \\ m \rightarrow 0}}=\omega^{\alpha} \cdot L_{\alpha}(0)=\omega^{\alpha} \neq 0 \quad \forall \alpha \in \mathbb{Z} .
$$

Therefore

$$
\begin{aligned}
W(0) & =\operatorname{det}\left[\left.\frac{d^{m} \omega_{l}^{\alpha} L_{\alpha}\left(\omega_{l}^{p} x\right)}{d x^{m}}\right|_{x \rightarrow 0}\right]_{l \times m} \\
& =\operatorname{det}\left[\omega_{l}^{p \cdot m}\right]_{l \times m} \cdot \prod_{l=1}^{n} \omega_{l}^{\alpha} \cdot \prod_{m=1}^{n-1}\left(\frac{\alpha}{n} \cdot\left(\frac{p \cdot m+\alpha-n}{n}\right)_{m-1}\right),
\end{aligned}
$$

where $m$ runs over $[n-1]_{0}$ and $l$ runs over $[n]$ in the determinant on the left in (12.3).

If $p$ and $n$ are relatively prime, $n \geq 3$, and $p \in[n-1]$, then $\operatorname{det}\left[\omega_{l}^{p \cdot m}\right]_{l \times m}= \pm$ $\operatorname{det}\left[\omega_{l}^{m}\right]_{l \times m}= \pm \sqrt{\chi}$, where $\chi \equiv(-1)^{n-1} n^{n}$ is the discriminant of $x^{n}-1$. Also, $\prod_{l=1}^{n} \omega_{l}^{\alpha}=$ $(-1)^{\alpha \cdot(n-1)}$. Furthermore, the product on the right in (12.3) equals

$$
\prod_{m=1}^{n-1}\left(\frac{p \cdot m+\alpha-n}{n}\right)_{m-1}=\prod_{m=2}^{n-1}\left(\frac{p \cdot m+\alpha-n}{n}\right)_{m-1}=\prod_{m=2}^{n-1} \prod_{k=0}^{m-2} \frac{p \cdot m+\alpha-n-k \cdot n}{n}
$$


or

$$
\prod_{m=2}^{n-1} \prod_{k=1}^{m-1} \frac{p \cdot m+\alpha-k \cdot n}{n}=n^{-\left(\frac{n-1}{2}\right)} \cdot \prod_{m=2}^{n-1} \prod_{k=1}^{m-1}(p \cdot m+\alpha-k \cdot n)
$$

Note that the first equality in (12.4) holds because $((p \cdot m+\alpha-n) / n)_{m-1}=((p+\alpha-$ $n) / n)_{0}=1$ when $m=1$ in the first product on the left in (12.4).

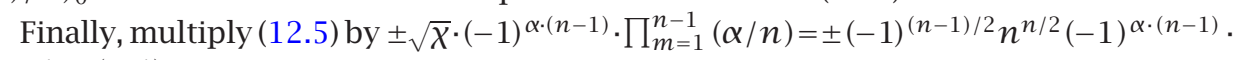
$\alpha^{n-1} n^{-(n-1)}$ to get

$$
W(0)= \pm(-1)^{(n-1) / 2} n^{n / 2}(-1)^{\alpha \cdot(n-1)} \cdot \alpha^{n-1} n^{-(n-1)} n^{-(n-1)} \prod_{m=2}^{n-1} \prod_{k=1}^{m-1}(p \cdot m+\alpha-k \cdot n)
$$

or

$$
W(0)= \pm(-1)^{(2 \alpha+1)(n-1) / 2} \cdot \alpha^{n-1} n^{-n(n-2) / 2} \cdot \prod_{m=2}^{n-1} \prod_{k=1}^{m-1}(p \cdot m+\alpha-k \cdot n) .
$$

Therefore, when $\operatorname{gcd}(p, n)=1, W(0)=0$ if and only if $\alpha \in\{0, k \cdot n-m \cdot p \ni k \in$ [m-1], $2 \leq m \leq n-1\}$, a set of at most $\left(n^{2}-3 n+4\right) / 2$ distinct integers. If $p$ and $n$ are not relatively prime, then $\operatorname{det}\left[\omega_{l}^{p \cdot m}\right]_{l \times m}=0$ implies $W(0)=0$, so the Wronskian is identically zero.

13. Computation of the Wronskian of the trinomial resolvent. If one wants the Wronskian at other points $x$ of the Cockle resolvent of $t^{n}+x \cdot t^{p}-1$, and $p$ and $\alpha$ satisfy the conditions of Theorem 12.4, one can use the Abel formula

$$
W(x)=W(0) \cdot \exp \left(-\int_{0}^{x} \frac{C_{n-1}(\zeta)}{\zeta \cdot C_{n}(\zeta)} \mathrm{d} \zeta\right)
$$

as noted in [11, page 119] to get $W(x)$.

EXAMPLE 13.1. For completeness, we compute the Wronskian $W(x)$ of Cockle's resolvent of the trinomial $z^{n}+x \cdot z^{p}-1=0$ using Abel's formula when $d=1$. It is easy to deduce that $C_{n}(x) \equiv 1$ from (11.3), (11.4), and (11.5) since the degree of $v$ in $f(v)$ is $n$. To compute $C_{n-1}(x)$, we need to compute $c_{n-1, f}=\left.\left(\tau^{n-1} f(v)\right)\right|_{v \rightarrow 0} \div(n-1)$ !. Since $\tau^{m} v^{k}=0=\tau^{m}(v)_{k}$ for any $k \in[m-1]_{0}$, we may ignore all powers of $v$ lower than $n-1$ when we apply the difference operator $\tau$ to any polynomial in $v$.

The coefficient of $v^{n-1}$ in $f(v)$ is just the negative of the first elementary symmetric function of the roots of $f(v)$

$$
\left\{-\frac{\alpha}{p}-i \cdot \frac{n}{p}\right\}_{i=0}^{p-1} \cup\left\{\frac{\alpha}{q}-i \cdot \frac{n}{q}\right\}_{j=0}^{q-1}
$$


which is just the negative of their sum

$$
\begin{aligned}
\sum_{i=0}^{p-1}\left(\frac{\alpha}{p}\right. & \left.+i \cdot \frac{n}{p}\right)+\sum_{j=0}^{q-1}\left(-\frac{\alpha}{q}+i \cdot \frac{n}{q}\right) \\
& =\alpha+\frac{n}{p} \cdot \frac{p(p-1)}{2}-\alpha+\frac{n}{q} \cdot \frac{q(q-1)}{2}=\frac{n \cdot(p+q)}{2}=\frac{n^{2}}{2} .
\end{aligned}
$$

The coefficient of $v^{n}$ in $f(v)$ is just 1 . Therefore, $c_{n-1, f}=\left.\left(\tau^{n-1}\left(v^{n}+n^{2} \cdot v^{n-1} / 2\right)\right)\right|_{v \rightarrow 0}$ $\div(n-1)$ !. Now $\left.\left(\tau^{n-1}\left(v^{n-1}\right)\right)\right|_{v \rightarrow 0} \div(n-1) !=1$ by [22, Problem 1.41, page 25], and $\left.\left(\tau^{n-1} \cdot\left(v^{n}\right)\right)\right|_{v \rightarrow 0} \div(n-1)$ ! is the Stirling number of the second kind $S_{n-1}^{n}$ in the notation in [22, formula (31), page 7] which equals $n \cdot(n-1) / 2$. One can determine this by [14, Example $11 \mathrm{~b}$, page 31 ], which says that $S_{n-1}^{n}$ equals the first complete symmetric function of the integers $[n-1]$, which is just their sum. So, $c_{n-1, f}=n \cdot(n-1) / 2+n^{2} / 2=$ $n \cdot(2 n-1) / 2$. Notice that $c_{n-1, f}>0$ for all $n \in \mathbb{N}$.

Substituting these expressions for $C_{n-1}(\zeta)$ and $C_{n}(\zeta)$ into Abel's formula (13.1) yields

$$
W(x)=W(0) \cdot \exp \left((-1)^{p+1} p^{p} q^{q} \frac{n \cdot(2 n-1)}{2} \int_{0}^{x} \frac{1}{\left((-p)^{p} q^{q} \zeta^{n}-n^{n}\right)} \zeta^{n-1} \mathrm{~d} \zeta\right)
$$

which can be integrated to

$$
W(x)=W(0) \cdot \exp \left(-\frac{(2 n-1)}{2} \ln \left(\frac{(-p)^{p} q^{q} x^{n}-n^{n}}{-n^{n}}\right)\right) .
$$

So

$$
W(x)=W(0) \cdot\left(\sqrt{1-(-p)^{p} q^{q} n^{-n} x^{n}}\right)^{-(2 n-1)}=W(0) \cdot \kappa \cdot(\sqrt{\Delta})^{-(2 n-1)},
$$

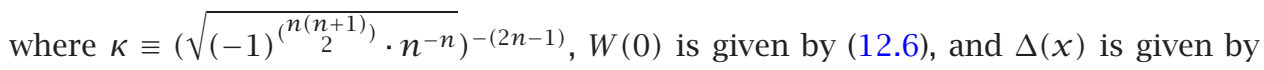
(11.22).

14. Determinantal formula. Finally, we prove that the only algebraic condition among the roots $\left\{z_{1}, \ldots, z_{n}\right\}$ of a polynomial $P$ which implies that there exists linear relations among the $n$ ! $n$ !-tuples of the form $\left(1, z_{1}, z_{1}^{2}, \ldots, z_{1}^{n-1}, z_{2}, z_{1} \cdot z_{2}, \ldots, z_{1}^{n-1}\right.$. $\left.z_{2}, \ldots, z_{1}^{n-1} \cdot z_{2}^{n-2} \cdots z_{n-1}^{1}\right)$ over $\mathbb{Q}$ is that two of the roots are equal.

LEMMA 14.1. Let $\left\{z_{1}, \ldots, z_{n}\right\}$ be a set of $n$ algebraically independent indeterminates over $\mathbb{Z}$. Let $G \approx S_{n}$ be the full symmetric group on $\left\{z_{1}, \ldots, z_{n}\right\}$. Let $I=\left(i_{1}, \ldots, i_{n}\right) \in \mathbb{N}_{0}^{n}$ be an $n$-tuple with $i_{k} \in[n-k]_{0}$, for all $k \in[n]$. Let $\mathrm{E}$ be the set of $n$ ! monomials in the polynomial ring $\mathbb{Z}\left[z_{1}, \ldots, z_{n}\right]$ of the form $\left\{z^{I} \equiv \prod_{k=1}^{n} z_{k}^{i_{k}}\right\}$. Let $M_{n}$ be the $n ! \times n !$ matrix $\left[g\left(t^{I}\right)\right]_{(g \in G) \times I}$. Let $d_{n} \equiv \operatorname{det}\left(M_{n}\right)$. Let $\Delta \equiv \prod_{i<j}\left(z_{i}-z_{j}\right)^{2}$. Then $d_{n}= \pm(\sqrt{\Delta})^{n ! / 2}$.

Proof. The term $d_{n}$ is a polynomial in $\left\{z_{1}, \ldots, z_{n}\right\}$ over $\mathbb{Z}$ of homogeneous degree. Therefore, either $d_{n}=0$ or the total degree in $\left\{z_{1}, \ldots, z_{n}\right\}$ of $d_{n}$ is just the sum of the degrees in $\left\{z_{1}, \ldots, z_{n}\right\}$ of $z^{I}$ over all $z^{I} \in \mathrm{E}$. We will next compute this degree. We will prove later that $d_{n} \neq 0$. 
Since $i_{1}$ takes on each value of $[n-1]_{0}$ exactly $n ! \div n$ times among all the monomials in E, and $i_{2}$ takes on each value of $[n-2]_{0}$ exactly $n ! \div(n-1)$ times among all the monomials in E,..., and $i_{n}$ takes on the value of 0 exactly $n ! \div 1$ times among all the monomials in $\mathrm{E}$, the total degree in $\left\{z_{1}, \ldots, z_{n}\right\}$ of $d_{n}$ is the sum

$$
\begin{aligned}
\frac{n !}{n} \cdot & \sum_{i_{1}=0}^{n-1} i_{1}+\frac{n !}{n-1} \cdot \sum_{i_{2}=0}^{n-2} i_{2}+\cdots+\frac{n !}{1} \cdot \sum_{i_{n}=0}^{0} i_{n} \\
& =n ! \cdot\left(\frac{1}{n} \frac{n(n-1)}{2}+\frac{1}{n-1} \frac{(n-1)(n-2)}{2}+\cdots+\frac{1}{1} \frac{1 \cdot 0}{2}\right) \\
& =\frac{n !}{2} \cdot \sum_{k=0}^{n-1} k=\frac{n !}{2} \cdot \frac{n(n-1)}{2} .
\end{aligned}
$$

The total degree in $\left\{z_{1}, \ldots, z_{n}\right\}$ of $\sqrt{\Delta}$ is $n \cdot(n-1) / 2$, so the total degree in $\left\{z_{1}, \ldots, z_{n}\right\}$ of $(\sqrt{\Delta})^{n ! / 2}$ is $(n ! / 2) \cdot(n \cdot(n-1) / 2)$.

Now we will prove that $(\sqrt{\Delta})^{n ! / 2}$ divides $d_{n}$ in the polynomial ring $\mathbb{Z}\left[z_{1}, \ldots, z_{n}\right]$. Pick any $u, v \in\left\{z_{1}, \ldots, z_{n}\right\}$ with $u \neq v$. Express $S_{n}$ as the disjoint union of the $n ! / 2$ cosets $C_{k}$ in $S_{n}$ of the subgroup $\{1,(u v)\}$. So, $S_{n}=\coprod_{k=1}^{n ! / 2} C_{k}$. Perform the following elementary row operations on $M_{n}$. Let $C_{k}=\left\{\theta_{k}, \sigma_{k}\right\}$, where $\sigma_{k}=(u v) \circ \theta_{k}$ for each $k \in[n ! / 2]$. Let $R \theta_{k}$ be the row $\left\{\theta_{k}\left(z^{I}\right)\right\}_{I \in \mathrm{E}}$ in $M_{n}$. Let $R \sigma_{k}$ be the row $\left\{\sigma_{k}\left(z^{I}\right)\right\}_{I \in \mathrm{E}}=\left\{\left((u v) \circ \theta_{k}\right)\left(z^{I}\right)\right\}_{I \in \mathrm{E}}=$ $\left\{(u v) \circ\left(\theta_{k}\left(z^{I}\right)\right)\right\}_{I \in \mathrm{E}}$ in $M_{n}$. Replace row $R \theta_{k}$ with $R \theta_{k}-R \sigma_{k}$. Since $(u-v)$ factors out of every entry in the row $R \theta_{k}-R \sigma_{k}$, and there are $n ! / 2$ such rows, we conclude that $(u-v)^{n ! / 2}$ factors out of $d_{n}=\operatorname{det}\left(M_{n}\right)$. Since the choice of $u, v \in\left\{z_{1}, \ldots, z_{n}\right\}$ was arbitrary, we conclude that $\prod_{i<j}\left(z_{i}-z_{j}\right)^{n ! / 2}$ divides $d_{n}$ in $\mathbb{Z}\left[z_{1}, \ldots, z_{n}\right]$.

We will now prove that $d_{n} \neq 0$ by induction on $n$. Assume the same definitions for $M_{n-1}$ and $d_{n-1} \equiv \operatorname{det} M_{n-1}$ as for $M_{n}$ and $d_{n}$ with $n-1$ replacing $n$ and $\left\{z_{1}, \ldots, z_{n-1}\right\}$ replacing $\left\{z_{1}, \ldots, z_{n}\right\}$ in the statement of Lemma 14.1. Assume that $d_{n-1} \neq 0$. It will be sufficient to show that the coefficient of the monomial $\pi \equiv \prod_{k=1}^{n} z_{k}^{(k-1) \cdot n ! / 2}$ in $d_{n}$ is not zero. The matrix $M_{n}$ has the following form. Exactly $(n-1)$ ! rows and $(n-1)$ ! columns have $(n-1)$ as the maximal power of $z_{n}$ among all their entries. Furthermore, the entries in these rows and columns possessing $z_{n}^{n-1}$ form an $(n-1) ! \times(n-1)$ ! submatrix $V_{n-1}$ of $M_{n}$ which is simply the matrix formed from $M_{n-1}$ by multiplying every entry in $M_{n-1}$ by $z_{n}^{n-1}$.

Repeat this procedure on the submatrix of $M_{n}$ formed by blocking out the submatrix $V_{n-1}$. Exactly $(n-1)$ ! rows and $(n-1)$ ! columns have $(n-2)$ as the maximal power of $z_{n}$ among all their entries. They form an $(n-1) ! \times(n-1)$ ! submatrix $V_{n-2}$ of $M_{n}$ which is simply the matrix formed from $M_{n-1}$ by multiplying every entry in $M_{n-1}$ by $z_{n}^{n-2}$.

Repeating this procedure, we create a finite sequence of $(n-1) ! \times(n-1)$ ! submatrices $\left\{V_{k}\right\}_{k=0}^{n-1}$ of $M_{n}$ with $v_{k} \equiv \operatorname{det}\left(V_{k}\right)=\left(z_{n}^{k}\right)^{(n-1) !} \cdot \operatorname{det}\left(M_{n-1}\right)=\left(z_{n}\right)^{k \cdot(n-1) !} \cdot d_{n-1} \neq 0$. The monomial $\pi$, possessing the highest degree in $z_{n}$ among all the monomials in $d_{n}$, can come only from

$$
\prod_{k=0}^{n-1} v_{k}=d_{n-1}^{n} \cdot z_{n}^{(n-1) ! \cdot \sum_{k=0}^{n-1} k}=d_{n-1}^{n} \cdot z_{n}^{(n-1) ! \cdot n((n-1) / 2)}=d_{n-1}^{n} \cdot z_{n}^{n ! \cdot((n-1) / 2)} \neq 0 .
$$

Therefore, the monomial $\pi$ appears with nonzero coefficient in $d_{n}$. So $d_{n} \neq 0$. 
EXAMPLE 14.2. Let $\{u, v, w\}$ be algebraically independent over $\mathbb{Z}$. Let $\mathrm{E}=\{1, u$, $\left.u^{2}, v, u v, u^{2} v\right\}$, where $u=z_{3}, v=z_{2}$, and $w=z_{1}$ as in Lemma 14.1. If $M_{3}=\left[g\left(z^{I}\right)\right]_{g \times I}$, then

$$
M_{3}=\left[\begin{array}{cccccc}
1 & u & u^{2} & v & u v & u^{2} v \\
1 & v & v^{2} & w & v w & v^{2} w \\
1 & w & w^{2} & u & w u & w^{2} u \\
1 & v & v^{2} & u & v u & v^{2} u \\
1 & w & w^{2} & v & w v & w^{2} v \\
1 & u & u^{2} & w & u w & u^{2} w
\end{array}\right]
$$

Rows 1 and 6 , respectively columns 3 and 6 , have 2 as the maximal power of $u$ in their entries. Among the entries in the $4 \times 4$ submatrix formed by deleting rows 1 and 6 and columns 3 and 6 , keeping the original numbering of rows and columns of the original $6 \times 6$ matrix $M_{3}$, rows 3 and 4 and columns 4 and 5 have 1 as the maximal power of $u$ in their entries. The $2 \times 2$ submatrix formed by deleting rows $1,3,4,6$ and columns $3,4,5,6$ has 0 as the maximal power of $u$ among its entries. We form the $(n-1) ! \times(n-1) !=2 \times 2$ submatrices $\left\{V_{k}\right\}_{k=0}^{2}$ in the following manner. Let $a_{i, j}$ denote the $(i, j)$ entry of $M_{3}$. Then

$$
\begin{aligned}
& V_{2}=\left[\begin{array}{ll}
a_{1,3} & a_{1,6} \\
a_{6,3} & a_{6,6}
\end{array}\right]=\left[\begin{array}{ll}
u^{2} & u^{2} v \\
u^{2} & u^{2} w
\end{array}\right], \\
& V_{1}=\left[\begin{array}{ll}
a_{3,4} & a_{3,5} \\
a_{4,4} & a_{4,5}
\end{array}\right]=\left[\begin{array}{ll}
u & w u \\
u & v u
\end{array}\right], \\
& V_{0}=\left[\begin{array}{ll}
a_{2,1} & a_{2,2} \\
a_{5,1} & a_{5,2}
\end{array}\right]=\left[\begin{array}{cc}
1 & v \\
1 & w
\end{array}\right] .
\end{aligned}
$$

Then $\operatorname{det}\left(V_{2}\right)= \pm u^{4} \cdot d_{2}$, $\operatorname{det}\left(V_{1}\right)= \pm u^{2} \cdot d_{2}$, and $\operatorname{det}\left(V_{0}\right)= \pm u^{0} \cdot d_{2}$, where $d_{2}=$ $\operatorname{det}\left(M_{2}\right)= \pm(v-w) \neq 0$ like the induction hypothesis used in the proof of Lemma 14.1. The only place that $u^{6}$ appears in $d_{3}$ is in the polynomial $\operatorname{det}\left(V_{0}\right) \cdot \operatorname{det}\left(V_{1}\right) \cdot \operatorname{det}\left(V_{2}\right)=$ $\pm u^{6}(v-w)^{3} \neq 0$. Therefore, $d_{3} \neq 0$.

To help visualize the fact that $(v-w)^{3}$ divides $d_{3}$ in the polynomial ring $\mathbb{Z}[u, v, w]$, perform the following elementary row operations on $M_{3}$. Replace $R_{1}$ with $R_{1}-R_{6}$, replace $R_{2}$ with $R_{2}-R_{5}$, and $R_{3}$ with $R_{3}-R_{4}$. Then the top three rows of $M_{3}$ become

$$
\left[\begin{array}{cccccc}
0 & 0 & 0 & (v-w) & u \cdot(v-w) & u^{2} \cdot(v-w) \\
0 & (v-w) & (v+w) \cdot(v-w) & -(v-w) & 0 & v w \cdot(v-w) \\
0 & -(v-w) & -(v+w) \cdot(v-w) & 0 & -u \cdot(v-w) & -u \cdot(v+w) \cdot(v-w)
\end{array}\right]
$$

each of which has $(v-w)$ as a factor.

15. Conclusions. Much work must be done to find formulae for the Cohnian of arbitrary, not just differentially transcendental, univariate polynomials over a differential field, in addition to determining their weight. The author's powersum formula [21] for 
a resolvent is guaranteed to yield a nonzero resolvent only for polynomials whose distinct roots are differentially independent by [19]. But the powersum formula has always worked for any polynomial the author has tested. Unfortunately, for differentially transcendental polynomials, the powersum formula yields a resolvent whose weight equals $\Psi \cdot(\Psi-1) / 2$, which is sextic in the number $n$ of distinct roots of the polynomial. Clearly, a huge amount of factoring must be done on this formula to recover the Cohnian. The author has made some progress in factoring some of the terms of an $\alpha$-resolvent of a differentially transcendental polynomial in [17].

\section{REFERENCES}

[1] G. Belardinelli, L'equazione differenziale risolvente dell'equazione trinomia, Rend. Circ. Mat. Palermo 46 (1922), 463-472 (Italian).

[2] A. Cayley, Note on a differential equation, Mem. Lit. Philos. Soc. Manchester 2 (1865), 111114.

[3] J. Cockle, Note on transcendental roots, Phil. Mag. 20 (1860), 369-373.

[4] _ Sketch of a theory of transcendental roots, Phil. Mag. 20 (1860), 145-148.

[5] _ On transcendental and algebraic solutions, Phil. Mag. 21 (1861), 379-383.

[6] On differential equations and on co-resolvents, Trans. Royal Soc. Victoria 7 (1865/1866), 176-191.

[7] On the explicit integration of certain differential resolvents, Proc. London Math. Soc. 14 (1883), 18-22.

[8] L. Comtet, Advanced Combinatorics. The Art of Finite and Infinite Expansions, revised \& enlarged ed., D. Reidel Publishing, Dordrecht, 1974, translated from French by J. W. Nienhuys.

[9] G. P. Egorychev, Integral Representation and the Computation of Combinatorial Sums, Translations of Mathematical Monographs, vol. 59, American Mathematical Society, Rhode Island, 1984.

[10] I. M. Gel'fand, M. M. Kapranov, and A. V. Zelevinsky, Discriminants, Resultants, and Multidimensional Determinants, Mathematics: Theory \& Applications, Birkhäuser Boston, Massachusetts, 1994.

[11] E. L. Ince, Ordinary Differential Equations, Dover Publications, New York, 1944.

[12] F. Kamber, Formules exprimant les valeurs des coefficients des séries de puissances inverses, Acta Math. 78 (1946), 193-204.

[13] E. R. Kolchin, Differential Algebra and Algebraic Groups, Pure and Applied Mathematics, vol. 54, Academic Press, New York, 1973.

[14] I. G. Macdonald, Symmetric Functions and Hall Polynomials, 2nd ed., Oxford Mathematical Monographs. Oxford Science Publications, Clarendon Press, Oxford University Press, New York, 1995.

[15] K. Mayr, Über die Lösung algebraischer Gleichungssysteme durch hypergeometrische Funktionen, Monatsh. Math. 45 (1937), 280-313 (German).

[16] H. Mellin, Resolution de l'equation algebrique generale a l'aide de la fonction gamma, Compte Rendus 172 (1921), 658-661 (French).

[17] J. M. Nahay, A partial factorization of the powersum formula, to appear in Int. J. Math. Math. Sci.

[18] _ Linear differential resolvents, Doctoral dissertation, Rutgers University, New Jersey, 2000.

[19]___ Powersum formula for polynomials whose distinct roots are differentially independent over constants, Int. J. Math. Math. Sci. 32 (2002), no. 12, 721-738.

[20] _ Linear relations among algebraic solutions of differential equations, J. Differential Equations 191 (2003), no. 2, 323-347. 
[21] _ Powersum formula for differential resolvents, Int. J. Math. Math. Sci. 2004 (2004), no. 7, 365-371.

[22] M. R. Spiegel, Calculus of Finite Differences and Difference Equations, McGraw-Hill, New York, 1994.

[23] B. Sturmfels, Solving algebraic equations in terms of A-hypergeometric series, Discrete Math. 210 (2000), no. 1-3, 171-181.

John Michael Nahay: 25 Chestnut Hill Lane, Columbus, NJ 08022-1039, USA

E-mail address: reso7vent@comcast.net 


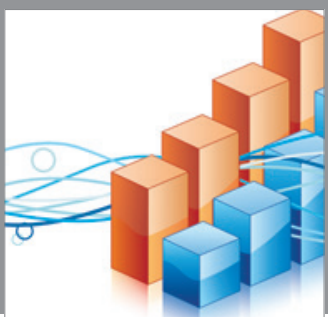

Advances in

Operations Research

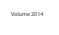

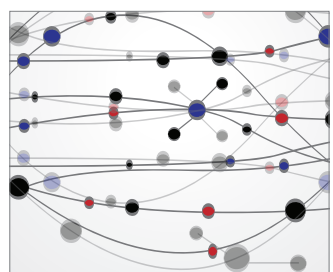

\section{The Scientific} World Journal
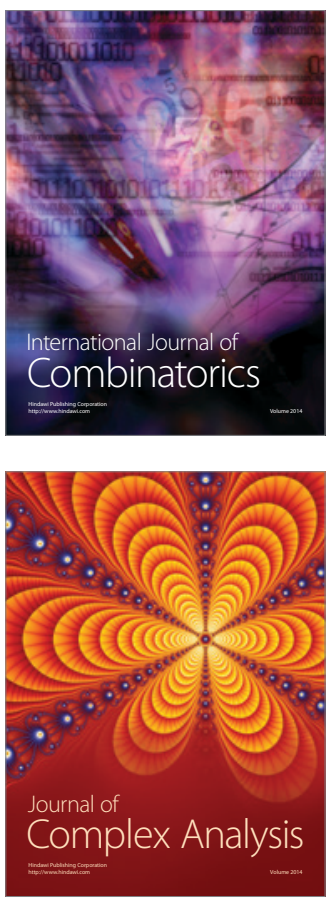

International Journal of

Mathematics and

Mathematical

Sciences
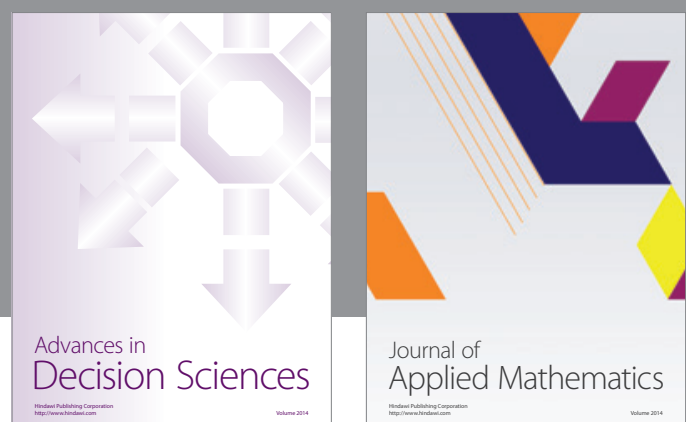

Journal of

Applied Mathematics
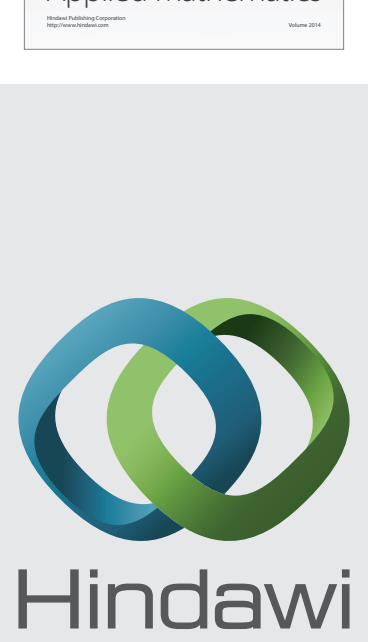

Submit your manuscripts at http://www.hindawi.com
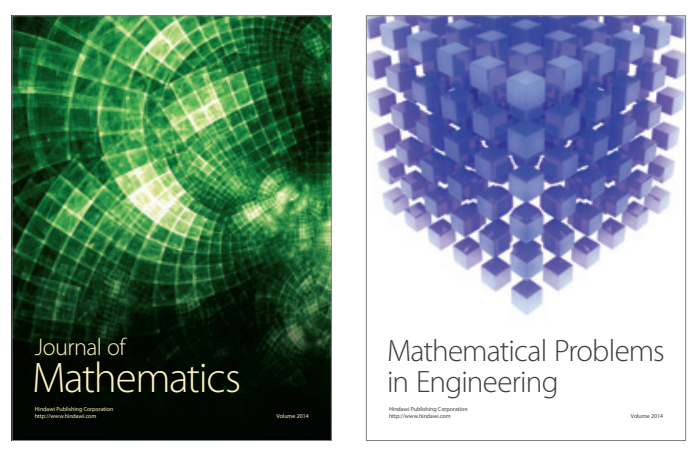

Mathematical Problems in Engineering
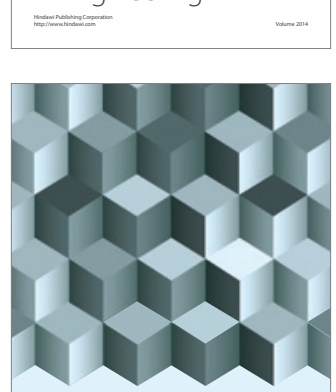

Journal of

Function Spaces
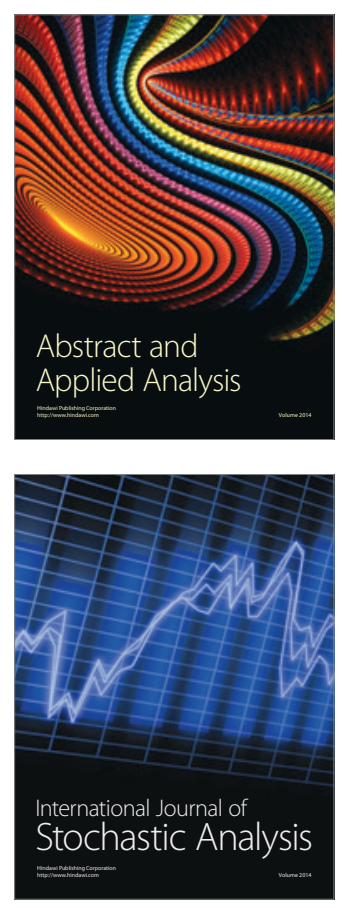

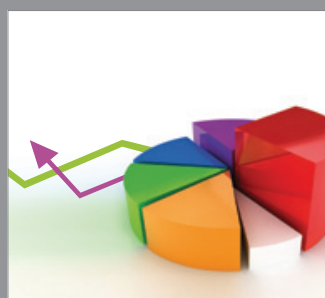

ournal of

Probability and Statistics

Promensencen
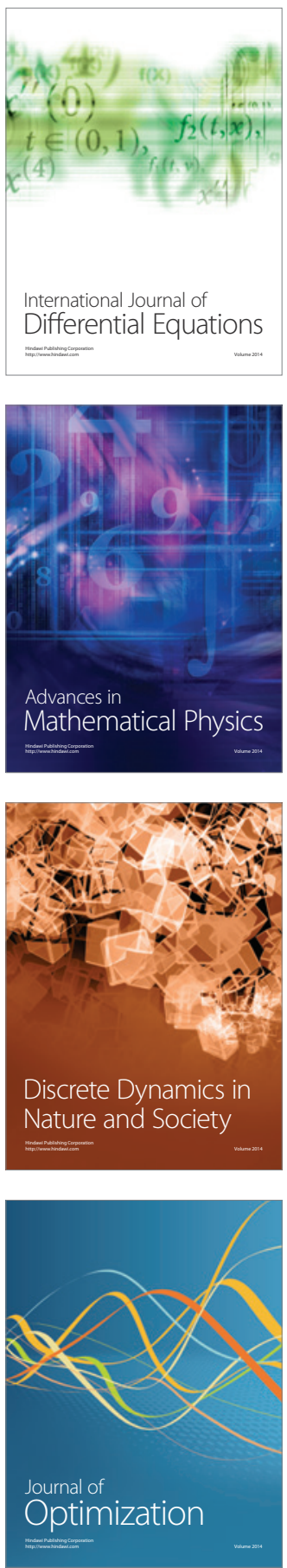